\title{
Terminal Distribution of the Corticospinal Projection from the Hand/Arm Region of the Primary Motor Cortex to the Cervical Enlargement in Rhesus Monkey
}

\author{
Robert J. Morecraft ${ }^{1}$, Jizhi Ge ${ }^{1}$, Kimberly S. Stilwell-Morecraft ${ }^{1}$, David W. McNeal ${ }^{1}$, Marc A. \\ Pizzimenti $^{2}$, and Warren G. Darling ${ }^{3}$ \\ ${ }^{1}$ Division of Basic Biomedical Sciences, Laboratory of Neurological Sciences, The University of \\ South Dakota, Sanford School of Medicine, Vermillion, South Dakota 57069. \\ ${ }^{2}$ Department of Anatomy and Cell Biology, Carver College of Medicine, The University of lowa, \\ lowa City, lowa, 52242. \\ ${ }^{3}$ Department of Health and Human Physiology, Motor Control Laboratories, The University of \\ lowa, lowa City, lowa 52242.
}

\section{Abstract}

To further our understanding of the corticospinal projection (CSP) from the hand/arm representation of the primary motor cortex (M1), high-resolution anterograde tracing methodology and stereology were used to investigate the terminal distribution of this connection at spinal levels C5 to T1. The highest number of labeled terminal boutons occurred contralaterally (98\%) with few ipsilaterally (2\%). Contralaterally, labeled boutons were located within laminae I - X, with the densest distribution found in lamina VII and, to a lesser extent, laminae IX and VI. Fewer terminals were found in other contralateral laminae. Within lamina VII, terminal boutons were most prominent in the dorsomedial, dorsolateral and ventrolateral subsectors. Within lamina IX, the heaviest terminal labeling was distributed dorsally. Ipsilaterally, boutons were found in laminae $\mathrm{V}-\mathrm{X}$. The most pronounced distribution occurred in the dorsomedial and ventromedial sectors of lamina VII and fewer labeled boutons were located in other ipsilateral laminae. Segmentally, contralateral lamina VII labeling was highest at levels C5-C7. In contrast, lamina IX labeling was highest at C7-T1 and more widely dispersed amongst the quadrants at C8-T1. Our findings suggest dominant contralateral influence of the M1 hand/arm CSP, a contralateral innervation pattern in lamina VII supporting Kuypers (1982) conceptual framework of a "lateral motor system", and a projection to lamina IX indicating significant influence on motoneurons innervating flexors acting on the shoulder and elbow rostrally (C5-C7), along with flexors, extensors, abductors and adductors acting on the digits, hand and wrist caudally (C8-T1).

ADDRESS CORRESPONDENCE: Dr. Robert J. Morecraft, Division of Basic Biomedical Sciences, Laboratory of Neurological Sciences, The University of South Dakota, Sanford School of Medicine, Vermillion, SD 57069, USA. (fax 605-677-6381; rmorecra@usd.edu).

Conflict of interest statement.

All authors do not have any conflict of interest that could inappropriately influence, or be perceived to influence, this work.

Role of authors. All authors listed on the paper contributed significantly to the elaboration of the paper and/or to the research that led to preparation of the manuscript. All authors had full access to all the data in the study and take responsibility for the integrity of the data and the accuracy of the data analysis. Study concept and design: RJM, WGD, MAP, DWM, KS-M, JG. Development of data: RJM, JG, KS-M, DWM. Acquisition of data: JG, RJM; Interpretation of data: RJM, WGD, MAP, KS-M, JG, DWM. Drafting of the manuscript: RJM, KS-M, WGD, MAP, JG, DWM. Obtained funding: RJM, WGD: Study supervision: RJM. 


\section{Keywords}

Cerebral Cortex; Frontal Lobe; Pyramidal Tract; Spinal Cord; Motor Control; Manual Dexterity; Hand Movement

\section{INTRODUCTION}

In the higher-order primate brain, the precentral cortex occupies the caudal-most part of the frontal lobe, just anterior to the central sulcus. In the late 1800's this brain region was inextricably linked to motor function when electrophysiological studies demonstrated that surface stimulation elicited contralateral peripheral movements (e.g., Ferrier, 1873; Horsley and Schäffer, 1888). In complementary fashion, surgical removal of the precentral gyrus in non-human primates resulted in severely impaired voluntary movement (e.g., Ferrier and Yeo, 1885; Horsley and Schäffer, 1888; Leyton and Sherrington, 1917). Collectively, these observations provided strong scientific support for the emerging clinical explanation of voluntary motor paresis following precentral damage in humans, in addition to addressing the longstanding question of cortical motor localization (Darling et al., 2011). Although selective removal of other frontal motor regions were later noted to give rise to detectable motor control deficits (e.g., Richter and Hines, 1932, 1934; Bucy, 1933; Fulton, 1937; Kennard and Fulton, 1933; Jacobsen, 1934; Travis, 1952, 1955), the more severe and debilitating acute consequences of precentral destruction in comparison, engendered the view that the precentral cortex may hold a pivotal, if not essential role in influencing fine motor control of the distal extremities in higher-order primates (for review see Darling et al, 2011; Wiesendanger, 2011).

Indeed, contemporary experimental studies in non-human primates have demonstrated that the primary motor cortex (M1) is involved in controlling skilled voluntary movements (Cheney et al., 1991; Porter and Lemon, 1993; Lemon et al., 2004; Martin, 2005; Lemon and Griffiths, 2005; Schieber, 2007). Microstimulation, single unit recording, and reversible inactivation studies show that M1 has considerable influence on shoulder and elbow movements to position the hand, and individuated digit movements governing opposition, grip formation and object manipulation (e.g., Evarts, 1968; Huntley and Jones, 1991; Georgopoulos et al., 1992; Maier et al., 1993; Schieber and Poliakov, 1998; Brochier et al. 1999; Park et al. 2001, 2004; Grandjean et al., 2007; Boudrias et al., 2010; Mollazadeh et al., 2011). Although M1 gives rise to a host of descending projections (e.g., Kuypers, 1981; Leichnetz, 1986; Kultas-Ilinsky et al., 2003; Schmahmann et al., 2004) it is well known that its corticospinal projection (CSP) represents an important structural substrate supporting these elements of skilled motor control (for review see Kuypers, 1981; Cheney et al. 1991; Lemon, 1993, 2008; Lemon et al., 2004, 2008; Schieber and Santello, 2004; Schieber, 2007). Furthermore, although it is firmly established in non-human primates that many cortical areas contribute to the CSP (e.g., Catsman-Berrevoets and Kuypers, 1976; Biber et al., 1978; Murray and Coulter, 1981; Nudo and Masterton, 1990; Dum and Strick, 1991; Galea and Darian-Smith, 1994), the CSP from M1 is the most prominent in terms of overall terminal density (Kuypers and Brinkman, 1970; Kuypers, 1981; Dum and Strick, 1996; Maier et al., 2002) and one of its most distinctive features is the substantial number of contralateral monosynaptic axonal projections to motoneurons residing in Rexed's lamina IX (e.g., Kuypers, 1960, 1981; Phillips and Porter, 1964; Fetz et al., 1989; Bortoff and Strick, 1993; Dum and Strick, 1996; Rouiller et al., 1996; Armand et al., 1997; Lemon et al., 2002; Maier et al., 1993, 2002; Boudrias et al., 2006, 2010a, 2010b). Corticomotoneuronal cells have been shown to be most abundant in the region of M1 lining the anterior bank of the central sulcus (Rathelot and Strick, 2006, 2009). Physiologically, the corticomotoneuronal projection is a unique characteristic of higher-order primates (Heffner 
and Masterton, 1975, 1983; Kuypers, 1981; Armand, 1982) that provides direct excitatory input to motoneurons (e.g., Lemon and Griffiths, 2005; Lemon, 2008; Schieber, 2007, 2011). It also appears to be unaffected by local spinal inhibitory mechanisms, and is more influential on distal intrinsic hand muscles compared to more proximal upper extremity muscles (for review see Porter and Lemon, 1993; Lemon et al., 2004; Lemon and Griffiths, 2005).

Currently, a great deal of neuroanatomical information has accumulated regarding the CSP from the upper extremity region of M1 in the non-human primate. It seems clear that the CSP from this region is predominately contralateral. However, there is a wide range of observations describing the proportion of contralateral versus ipsilateral projections. For example, a densitometric evaluation of wheat germ agglutinin-horseradish peroxidase (WGA-HRP) reaction product in the spinal gray matter following its injection into the arm area of M1 demonstrated that $82 \%$ of the CSP ends contralaterally with $18 \%$ ending ipsilaterally (Dum and Strick, 1996). In contrast, a recent study averaging the total number of biotinylated dextran amine (BDA) labeled axon fiber "elements" in the lateral corticospinal tract (LCST) indicated that $85-98 \%$ of the M1 hand/arm projection courses contralaterally (Yoshino-Saito et al., 2010) whereas another study using a similar approach estimated the contralateral projection to be in the 94-95\% range (Rouiller et al., 1996). These differences may be significant in terms of interpreting ipsilateral contributions to hand/arm motor function, as well as after unilateral damage to M1 because the capability of the undamaged, contralesional M1 to provide control over the ipsilateral limbs may be functionally related to the original number, and topographical location of ipsilateral termination. Since labeled axon number in the LCST may not be related to actual terminal bouton number in the spinal gray matter, and the densitometric method quantifies both labeled gray matter axons and terminals, an alternative approach to gain insight into the laterality of this projection would be to investigate the proportion of labeled contralateral and ipsilateral terminal boutons using the high resolution tract tracing methods currently available. Furthermore, estimating terminal boutons in the spinal gray matter may provide greater insight into the functional influence of this projection while providing a more direct structural correlate for drawing comparisons with physiological observations obtained in the monkey model.

In terms of laminar distribution, a number of tract tracing studies have shown that CSP terminals from the hand/arm region of M1 in the macaque monkey end in Rexed's laminae I - X contralaterally, and in laminae V - X ipsilaterally (Ralston and Ralston, 1985;

Leichnetz, 1986; Dum and Strick, 1996; Rouiller et al., 1996; Armand et al., 1997; Maier et al., 2002; Rosenzweig et al., 2009; Yoshino-Saito et al., 2010). The most prominent target is contralateral lamina VII (Liu and Chambers, 1964; Kuypers and Brinkman, 1970; Coulter and Jones, 1977; Ralston and Ralston, 1985; Leichnetz, 1986; Armand et al., 1994, 1997; Dum and Strick, 1996; Maier et al., 2002; Yoshino-Saito et al., 2010) followed by a fairly robust, but less dense projection to lamina IX (Ralston and Ralston, 1985; Leichnetz, 1986; Dum and Strick, 1996; Armand et al., 1997; Maier et al., 2002; Yoshino-Saito et al., 2010). However, the anatomical evidence suggests that the topographical distribution of the terminal projection within each lamina may not be uniform. For example, the M1 hand/arm projection appears to heavily innervate the dorsolateral part of lamina VII (Kuypers and Brinkman, 1970; Coulter and Jones, 1977; Kucera and Wiesendanger, 1985; Ralston and Ralston, 1985; Dum and Strick, 1996; Rouiller et al., 1996; Armand et al., 1994, 1997; Maier et al., 2002; Yoshino-Saito et al., 2010). Dense labeling has also been observed in the ventrolateral (Kuypers and Brinkman, 1970; Dum and Strick, 1996; Armand et al., 1994, 1997; Maier et al., 2002), dorsomedial (Kuypers and Brinkman, 1970; Armand et al., 1997) and ventromedial (Dum and Strick, 1996) regions of lamina VII. In addition to reexamining this issue in the context of the uncertainties noted, further experimental analysis of this 
organization with contemporary tract tracers should be of great interest in light of the recent attention being focused on understanding the functional contribution of monkey spinal interneurons and propriospinal neurons in the control of arm and hand movements (e.g., Alstermark et al., 1999; 2011; Fetz et al., 2002; Isa et al., 2006; Moritz et al., 2007; Takei and Seki, 2010; Kinoshita et al., 2012; Wu and Perlmutter, 2013; Zinger et al., 2013). With respect to the contralateral projection to lamina IX, there is substantial evidence to indicate heavy input to the dorsolateral region (Kuypers and Brinkman, 1970; Ralston and Ralston, 1985; Leichnetz, 1986; Armand et al., 1997; Dum and Strick, 1996; Rouiller et al., 1996; Maier et al., 2002; Yoshino-Saito et al., 2010) and some evidence indicating a strong projection to the dorsomedial (Kuypers and Brinkman, 1970; Leichnetz, 1986; Rouiller et al., 1996; Armand et al., 1997; Maier et al., 2002) and ventrolateral subsectors (YoshinoSaito et al., 2010). In this context, it is noteworthy that there are variable accounts of the contralateral projection to lamina VIII (Dum and Strick, 1996; Rouiller et al., 1996; Armand et al., 1997; Maier et al., 2002; Yoshino-Saito et al., 2010). A fuller description of these connectional patterns using high-resolution tract tracers may provide a better understanding of clinical complications such as spasticity and abnormal movement synergies observed after brain damage affecting M1 (Dewald et al. 1999).

To further our understanding on the laterality and terminal connectivity of the CSP from the hand/arm region of M1, we investigated the terminal distribution of this important projection to spinal segmental levels innervating the upper extremity (C5-T1) using contemporary high-resolution dextran tract tracing techniques in combination with intracortical microstimulation and stereological methodology in the rhesus monkey (Macaca mulatta). By its very nature, these tract tracers allow for light-level microscopic distinction between terminal axon fibers and terminal boutons of which the latter are reliable estimators of putative excitatory synaptic contacts (e.g., Wouterlood and Groenewegen, 1985; Freese and Amaral, 2006). This application, in conjunction with the use of design-based stereological quantification (West, 2012), allows for an unbiased estimation of immunohistochemically labeled terminal-like boutons in a relatively large biological tissue sample. Since previous studies suggest that the CSP to some laminae may be topographically specific as indicated above, we subdivided laminae I - X on each side of the spinal gray matter into 20 distinct regions of interest for our stereological analysis.

\section{MATERIALS AND METHODS}

The terminal distribution of the corticospinal projection arising from the hand/arm representation of M1 at spinal levels C5-T1 was studied in four rhesus monkeys (Macaca mulatta) (Figs. 1, 2; Table 1). All experimental and surgical procedures used in this study followed United States Department of Agriculture, National Institutes of Health, and Society for Neuroscience guidelines for the ethical treatment of animals and were approved by the Institutional Animal Care and Use Committee at The University of South Dakota. Details of the experimental methods used to accomplish the goals of this study are provided below.

\section{Neurosurgical Exposure, Intracortical Microstimulation and Tract Tracer Injection}

All neurosurgical procedures were performed using sterile methods. Preoperatively, each monkey was immobilized with atropine $(0.5 \mathrm{mg} / \mathrm{kg})$ then ketamine hydrochloride $(10 \mathrm{mg} / \mathrm{kg})$. The monkey was then intubated, placed on a mechanical ventilator and anesthetized with a mixture of 1.0-1.5\% isoflurane and surgical grade air/oxygen. The monkey was subsequently placed into a neurosurgical head holder and administered mannitol intravenously $(1.0-1.5 \mathrm{~g} / \mathrm{kg})$. For surgical exposure of the lateral frontal cortex, a skin incision was made followed by a frontoparietal craniotomy. Briefly, a bone flap was created over the pericentral cortex followed by dural opening centered over the central sulcus. 
Using electrophysiological microstimulation, and a combination of ketamine $(10 \mathrm{mg} / \mathrm{kg})$ and diazepam $(1.0 \mathrm{mg} / \mathrm{kg}$ ), the hand/arm representation of the primary motor cortex (M1 or area 4) in one randomly chosen hemisphere was localized prior to making the tract tracer injection (Fig. 1). The stimulation procedure included localizing the leg representation and the shoulder representation boundary dorsally, and the face boundary ventrally. Stimulation was performed using a Grass Square Pulse Stimulator system (model S28; Grass

Technologies, West Warwick, RI) with an attached tungsten electrode (impedance 0.5-1.5 $\mathrm{M} \Omega$ ). The electrode was secured in an electrode micromanipulator unit (Kopf Instruments, Model 1760-61, Tujunga, CA) and was placed $100 \mu \mathrm{m}$ below the pial surface and then advanced at $500 \mu \mathrm{m}$ intervals. Movements were evoked using a train duration of $50 \mathrm{~ms}$ and pulse duration of $0.2 \mathrm{~ms}$ delivered at $330 \mathrm{~Hz}$. Current intensity ranged between 1 and $90 \mu \mathrm{A}$. Threshold currents were determined and the evoked movements were recorded if noted by 2 observers. Each stimulation site location was recorded for reconstruction purposes. For accuracy, the recorded position of each electrode was made relative to general features of the surgical exposure in addition to gyral and sulcal landmarks and surface vessels.

Electrode penetration sites were intentionally minimized to avoid gray matter tissue damage while maximizing optimal tract tracer uptake and transport from corticospinal projection neurons.

After defining the physiological borders of the hand/arm representation in M1, tract tracer injections were placed into the metrically determined center of the hand/arm representation along the medial-lateral axis of the central sulcus (Fig. 1). Three syringe penetrations were made in a triangular pattern $1.5 \mathrm{~mm}$ apart from each other (Figure 1, see needle penetration points). The injections were made using a surgical microscope and Hamilton microsyringe that was secured in a specially designed Hamilton microdrive attached to the Kopf micromanipulator unit. For the first 2 injections the needle was placed perpendicular to the cortical surface, close to, but avoiding contact with the Rolandic artery. The needle was then angled to assume a slight rostral tilt to target layer $\mathrm{V}$ which widens in the anterior bank (Morecraft et al., 2012) and harbors the corticospinal projection neurons (Figure 2D, note slight angle of injection site core). After positioning, the needle was slowly advanced 3.5$4.5 \mathrm{~mm}$ deep within the cortex lining the depth of the anterior bank of the central sulcus. For the third penetration the needle was placed perpendicular to the gyral surface in a location between the first 2 penetrations but $1.5 \mathrm{~mm}$ rostral to the anterior edge of the central sulcus. Following positioning, the needle was carefully advanced $3-3.5 \mathrm{~mm}$ below the pia mater. In all experimental cases, $0.4 \mu \mathrm{L}$ of tracer was deposited in each penetration (totaling $1.2 \mu \mathrm{L}$ per case) (Table 1).

Following the injection procedure, the surgical field was irrigated with $0.9 \%$ sterile saline then gently swabbed. The dura was repositioned, closed with sutures and the bone flap replaced and anchored. To conclude the surgery, the temporalis muscle was sutured in place and the skin was closed using standard surgical technique. The animal was monitored and Bicillin L-A was used as pre- and post-operative prophylaxis antibiotic and buprenorphine $(0.01 \mathrm{mg} / \mathrm{kg})$ was used as a post-operative analgesic.

\section{Tissue Processing}

Following a survival period of 32-33 days after tract tracer injection, each monkey was deeply anesthetized with an overdose of pentobarbital $(50 \mathrm{mg} / \mathrm{kg}$ or more) and perfused transcardially with $0.9 \%$ saline. Saline infusion was followed by 2 liters of $4 \%$ paraformaldehyde in $0.1 \mathrm{M}$ phosphate buffer at $\mathrm{pH} 7.4(\mathrm{~PB})$ to fix the tissue, then one liter each of $10 \%$ and $30 \%$ sucrose in $0.1 \mathrm{M} \mathrm{PB}$ for tissue cryoprotection. The central nervous system was removed, placed in $30 \%$ sucrose in $0.1 \mathrm{M} \mathrm{PB}$ and stored for 2 to 5 days at $4^{\circ} \mathrm{C}$. During removal of the spinal cord region, several millimeters of each spinal root were intentionally left on the cord. Prior to blocking the tissue into manageable lengths for 
microtome sectioning, the cortex, brainstem and spinal cord were photographed with metric calibration. Ventral and dorsal roots of the spinal cord were extended so their positions relative to the long axis of the cord could be clearly localized. In addition to the use of microscopy, this information assisted in determining the segmental level locations and intersegment boundaries during the data analysis and reconstruction process.

In all cases, the cerebral cortex was frozen sectioned in the coronal plane on a sliding microtome (American Optical 860, Buffalo, NY, USA) at a thickness of $50 \mu \mathrm{m}$ in cycles of 10 , forming 10 complete series of evenly spaced tissue sections respectively. Each spinal cord was blocked from the central nervous system, frozen with dry ice and cut horizontally on the sliding microtome at a thickness of $50 \mu \mathrm{m}$ in cycles of 6 or 8 , with each forming a complete series of evenly spaced tissue sections respectively. For both the cortical and spinal cord sections, one series of tissue sections was mounted on subbed slides, dried and eventually stained for Nissl substance using thionin to evaluate cytoarchitectonic organization (Morecraft et al., 1992, 2004, 2012).

In all monkeys involved in this study, BDA was injected into a cortical region of interest as was LYD. Thus, subsequent series of tissue sections through the cortex and spinal cord were then processed using single (BDA alone) and double label (BDA + LYD) immunohistochemical procedures for localization of the neural tracers (Morecraft et al., 2007a). With respect to the tracer experiments used for the current study, LYD was injected into the hand/arm region of M1 in 3 animals and BDA was injected into the hand/arm region of M1 in one animal (Figs. 1, 2; Table 1).

To accomplish our objectives, one series of tissue sections from the cortex and spinal cord was used to process BDA alone (single labeling procedure) using the Vectastain Elite avidin-biotin complex $(\mathrm{ABC})$ labeling procedure (PK-6100 Kit, Vector Laboratories, Burlingame, CA, USA) (Figs. 1, 2D, 3D). Briefly, the tissue sections were rinsed in $0.05 \mathrm{M}$ tris buffered saline at $\mathrm{pH} 7.4$ (TBS) then incubated overnight in TBS with 5\% normal goat serum (NGS) and $1.25 \%$ Triton X-100. Next, the sections were rinsed in TBS and then incubated in the $\mathrm{ABC}$ solution for 4 hours at room temperature. The sections were then rinsed with TBS and incubated in a $0.05 \%$ solution of 3, 3' diaminobenzidine tetrahydrochloride (DAB) without nickel enhancement for approximately 10 minutes. Subsequently, $30 \%$ hydrogen peroxide $\left(\mathrm{H}_{2} \mathrm{O}_{2}\right)$ was added to the DAB solution achieving a final $\mathrm{H}_{2} \mathrm{O}_{2}$ concentration of $0.012 \%$. The tissue was incubated in the $\mathrm{DAB} / \mathrm{H}_{2} \mathrm{O}_{2}$ solution for another 8-10 minutes yielding an insoluble brown reaction product and immediately placed in TBS to stop the reaction. Following immunohistochemical processing, the BDA stained tissue sections were then rinsed in TBS, mounted on subbed slides, dried, then dehydrated in graded alcohol solutions and coverslipped using Permount. Next, an additional separate series of tissue sections was used for double labeling immunohistochemistry in which both BDA and LYD were visualized employing a simple multiple colorimetric detection method (Fig. 3A, B, C). To accomplish this, BDA was reacted first in a full series of tissue sections according to the above protocol staining BDA brown. The same tissue sections were then rinsed in TBS and incubated in TBS with 5\% NGS and $1.25 \%$ Triton X-100 overnight. The tissue sections were then transferred and incubated in 5\% goat serum in TBS with biotinylated anti-lucifer yellow directed against LYD at a dilution of 1:200 (anti-lucifer yellow, rabbit IgG fraction, biotin-XX conjugate, A5751, Molecular Probes, Eugene, OR) for approximately 40 hours at $4^{\circ} \mathrm{C}$. The tissue was then rinsed in TBS and incubated in a solution of $\mathrm{ABC}$ for 4 hours at room temperature, rinsed again in TBS and incubated with the Vector SG peroxidase substrate kit (Vector SK-4700, Vector laboratories, Burlingame, CA, USA) for approximately 5-10 minutes yielding a blue reaction product for the second tracer (LYD) (Figs. 2, 3). The sections were 
rinsed, mounted on subbed glass slides, dried, dehydrated and coverslipped using Permount. Thus, BDA was stained brown and LYD was stained blue in the same tissue sections.

To verify that the LYD antibody and subsequent tissue labeling process resulted in staining only the injected and transported tract tracer, sections from the rostral prefrontal cortex and caudal occipital lobe were immunohistochemically processed because these cortical regions are not connected to M1 (Leichnetz, 1986). The same analysis was performed to evaluate potentially false BDA labeling. In all 4 monkey cases, neuronal immunohistochemical labeling was not found in these control tissue sections.

\section{NeuN Staining Procedure}

In 2 additional animals that did not receive motor cortex injections, we studied NeuN stained spinal cord sections that were cut in the horizontal plane at $50 \mu \mathrm{m}$ in cycles of 6 . Although the Nissl and NeuN staining methods are comparable, an advantage of the NeuN method is that it selectively stains only nerve cells (Mullen et al., 1992; Wolf et al., 1996) in contrast to the Nissl method that stains nucleic acids, and thus Nissl substance of neurons, glial and endothelial cells. To accomplish this procedure, each animal was perfused with $0.9 \%$ saline and $4 \%$ paraformaldehyde in $0.1 \mathrm{M}$ phosphate buffer followed by sucrose cryoprotection and tissue sectioning as described above. Then one complete set of tissue sections was rinsed in $0.05 \mathrm{M}$ TBS adjusted to a $\mathrm{pH}$ of 7.4 (TBS). The tissue was then incubated in TBS with 5\% normal goat serum (NGS) and $1.25 \%$ triton $\mathrm{x}-100$ overnight at $4^{\circ} \mathrm{C}$. Next, the sections were incubated in 5\% NGS in TBS with mouse anti-neuronal nuclei (NeuN) monoclonal antibody (MAB 377, lot 0604027006, Chemicon, Temecula, CA) at a dilution of 1:1000 overnight at $4^{\circ} \mathrm{C}$ (Morecraft et al., 2012). Following rinsing in TBS, the tissue sections were incubated in biotinylated anti-mouse IgG (BA-9200, Vector Laboratories, Burlingame, CA) at a dilution of 1:500 at room temperature for three hours then rinsed in TBS. All sections were then incubated in a solution of $\mathrm{ABC}$ for 3 hours at room temperature, rinsed again in TBS, and incubated with the vector SG peroxidase substrate kit for approximately 2-10 minutes, yielding a blue reaction product. The tissue sections were rinsed in TBS, mounted on subbed slides then dried at room temperature. Finally, the sections were dehydrated in graded levels of increased concentrations of alcohol, cleared in xylene, then coverslipped using Permount.

\section{Definition of Anatomical Terminology}

To evaluate the distribution of terminal labeling, three major anatomical subdivisions of the spinal gray were recognized including the dorsal horn, intermediate zone, and ventral horn (Fig. 4A). For the present report, the dorsal horn refers to laminae I-IV, the intermediate zone is comprised of laminae V-VIII, and the ventral horn contains lamina IX (Figs. 4, 5) (Kuypers, 1981). Lamina X, originally described by Rexed (1954) as a central gray area (substantia grisea centralis and substantia gelatinosa centralis), surrounds the central canal and is considered its own midline area. In the intermediate zone, a distinct area lateral to laminae V, VI and VII was delineated and termed the reticulated marginal border (RMB) (McNeal et al., 2010). This area was recognized by Rexed (1954) and Kuypers (1981) as possessing extensive dendritic arbors of neurons located in the lateral part of laminae V-VII that extend into the dorsolateral funiculus. This primarily white matter area is a prominent target for terminal projections from motor cortex (McNeal et al., 2010).

For our stereological analysis, we subdivided laminae I-VI into medial and lateral halves, laminae VII into five subsectors (dorsolateral, ventrolateral, dorsomedial, ventromedial, and ventral) and lamina IX into quadrants (dorsolateral, ventrolateral, dorsomedial, ventromedial) based upon the general musculotopic organization of non-human primate motoneurons (Jenny and Inukai, 1983) (Fig. 4B). Specifically, it has been shown that neurons innervating flexors are commonly located dorsally in lamina IX and neurons 
innervating the extensors are frequently located more ventrally (Jenny and Inukai, 1983). Similarly, motoneurons located medially in lamina IX typically innervate more proximal musculature whereas neurons located more laterally have been found to innervate the distal musculature. Lamina $\mathrm{X}$ was divided into a contralateral half (Xc) and an ipsilateral half (Xi). In total, there were 20 stereological regions of interest (ROI) in the contralateral spinal cord (C5-T1) and 20 ROI's in the ipsilateral spinal cord (C5-T1).

\section{Data Analysis}

Localization of the cortical injection site (within the hand/arm area of M1) (Fig. 2) and the terminal boutons within the spinal gray matter and reticulated marginal border (C5-T1) (Fig. 3) was accomplished using brightfield illumination on a BX-51 Olympus microscope (Leeds Precision Instruments, Minneapolis, MN). Attached to the microscope was a high resolution MAC 5000 motorized stage (Ludl Electronic Products, Hawthorne, NY, USA) which was joined to the Neurolucida and Stereo Investigator neuroanatomical data collection software (Microbrightfield, Colchester, VT, USA) in a Dell Optiplex GX 280. The Neurolucida system was used to plot the major anatomical structures and their boundaries in Nissl and immunohistochemical stained tissue sections and record the locations of the injection site and terminal-like profiles (boutons) in the immunohistochemically processed tissue sections.

The injection sites were localized by plotting the external boundary of the core region and external boundary of the halo region (Fig. 2). The core region of the injection site was defined as the location of dense immunohistochemically reaction product characterized microscopically by a dense brown-black appearance in BDA sections and a dense blue-black appearance in LYD material obscuring cellular detail of the gray matter (Mesulam, 1982). The zone characterized as internal limit of the halo was defined within the limits of the gray matter (i.e., from layer I to the bottom of layer VI) where the dense precipitate that characterized the core zone diminished (Mesulam, 1982). The external limit of the halo was defined where small grains of reaction product were lightly interspersed among anterogradely labeled axons and terminal boutons, and well-defined retrogradely labeled cell bodies. Matching Nissl stained tissue sections were used to assist in the cytoarchitectonic and laminar analysis of the cortex containing the injection site.

In immunohistochemically processed spinal cord sections, terminal boutons were plotted in every other tissue section to obtain a general characterization of the topography and relative density of the projection in Rexed's laminae using Olympus UPlanApo 20x-40x microscope objectives (Leeds Precision Instruments, Minneapolis, MN). Matching Nissl stained sections were used to define laminar compartmentalization of the gray matter as well as the segmental level boundaries (Figs. 4, 5, 6, 7 and 8). NeuN stained tissue sections generated in two additional animals (not used in the tract tracing experiments presented in this report) were consulted to assist in defining the laminar sub-compartments and segmental levels boundaries (Fig. 5). As will be detailed below, in three monkey cases, the same sections (every other section through the entire C5 to T1 series) were then used to estimate bouton number in Rexed's laminae using stereology. Immunoreactive terminal-like varicosities (i.e., putative boutons or terminal-like profiles) (e.g., see insets in Fig. 3) were defined as small swellings along the terminal fibers that were $0.5-3.5 \mu \mathrm{m}$ in diameter (Lawrence et al., 1985; Wouterlood and Groenewegen, 1985; Freese and Amaral, 2006; Morecraft et al., 2007b). In our stereological analysis, microscopic identification of all boutons was accomplished using an Olympus PlanApo 100x oil objective (Leeds Precision Instruments, Minneapolis, MN). 


\section{Stereological Data Analysis}

Using Stereo Investigator 7 (MicroBrightField Inc. Williston VT, USA), unbiased estimates of terminal boutons were obtained in the spinal gray matter ROI's using the Optical Fractionator probe. An estimation of the cortical injection site volume (halo and core) was achieved by applying the Cavalieri probe. We designed and constructed the parameters of the probes according to our recently published material (Morecraft et al., 2007b; McNeal et al., 2010). Briefly, the stereological parameters included the counting brick dimensions, tissue thickness, counting brick placement, guard zones and disector height. The same counting frame $(109.2 / 71.4 \mu \mathrm{m})$ and $\mathrm{X} / \mathrm{Y}$ grid placement $(125.3 / 241.9 \mu \mathrm{m})$ was applied to all case material (40 total ROI's, 20 per side in every other tissue section) when performing stereology. Tissue thickness was determined by sampling 5 random gray matter sites in every other section then computing an average from this data.

The Optical Fractionator is a three-dimensional stereological probe facilitating microscopic estimation of population sizes. This is achieved by counting objects with optical disectors in a systematic sample comprising a known fraction of the total volumes of the region under analysis (Gundersen et al., 1988; Glasser et al., 2007; West, 2012). The counting target consisted of immunoreactive terminal-like varicosities that are $0.5-3.5 \mu \mathrm{m}$ in diameter (Fig. 3) which were counted using unbiased counting rules. It is important to note that as determined by electron microscopic analysis, varicosities of this size on the terminal region of axon fibers that are immunohistochemically identified with the use of this type of anterograde tracer primarily represent excitatory synaptic contacts with local neurons (i.e., > 98\%) (Wouterlood and Groenewegen, 1985; Freese and Amaral, 2006). Indeed, the goal of this study was not to investigate the microsynaptic organization of the M1 corticospinal projection but to derive an estimate of axon innervation in a large tissue sample based upon the exquisite structural detail of tracer labeled terminal-like swellings obtained from the immunohistochemical procedures applied in the study.

Additionally, estimates of the volume of tissue that constituted the core and halo of each injection site were accomplished with the Cavalieri Probe (Table 1) (Pizzimenti et al., 2007). The computed value representing the halo volume included the core volume. To accomplish this analysis, a contour was drawn around the ROI (e.g., the peripheral boundary of the core region or the peripheral boundary of the halo region) in every tissue section containing the injection site (Fig. 2). The probe then placed a rectangular grid consisting of points separated by defined distances over the ROI. An unbiased estimation of the overall injection site volume (halo) was calculated using Stereo Investigator software by factoring in tissue section thickness, the area of the ROI, the number of sections containing the injection site ROI's, and the distance between the points within the grid array. An unbiased estimation for the injection site core volume was accomplished in the same manner.

\section{Data Reconstruction and Presentation}

Publication quality images of injection sites and labeled fibers were captured using a Spotflex 64 Mp shifting pixel camera, (Diagnostic Instruments Inc., Sterling Heights, MI, USA, version 4.6), mounted on an Olympus BX51 microscope. Photographic montages of the injection sites and labeled fibers were created using Adobe PhotoShop 7.0 (Adobe Systems Inc., San Jose, CA, USA). Brightness and contrast were adjusted in the images. Cortical reconstructions were developed as previously described using metrically calibrated digital images of the cortical surface (Morecraft and Van Hoesen, 1992, 1993). Publication quality illustrations were created using Slidewrite 6.0 for data graphs (Rancho Santa Fe, CA, USA) and Adobe Illustrator 10.0 for line drawings (Adobe Systems Inc., San Jose, CA, USA). 


\section{RESULTS}

Histological analysis of all 4 experimental cases revealed that all tract tracer injection sites were confined to area 4 of the precentral cortex (Fig. 1). In no circumstance did the tracer spread into the fundus and posterior bank of the central sulcus to involve areas 3,1 and 2 of the somatosensory cortex or involve area 6 of the premotor cortex on the lateral surface (Fig. 2). All injection sites involved layer $V$ of area 4 , which harbors pyramidal cells that give rise to corticospinal projections. The injection sites in cases SDM54, SDM61 and SDM62 all involved the depths of the anterior bank of the central sulcus including the adjacent cortex on the gyral convexity (Figs. 1, 2A, C, D). All three injection sites resulted in dense axon and terminal labeling throughout spinal levels C5 to T1 (Figs. 6-8). In contrast, the injection site in case SDM57 involved primarily the gyral convexity with less involvement of layer V and little involvement of the cortex lining the anterior bank of the central sulcus (Figs. 1, 2B). As a result, this injection site produced much weaker labeling at spinal levels C5 to T1. Thus, cases SDM54, SDM61 and SDM62 were used for stereological analysis of bouton distribution. Case SDM57 was used to verify the general topographical labeling patterns found in the stereological analysis.

The estimated numbers of labeled terminal boutons within each ROI (with the exception of the subsector breakdown for labeling in laminae VII and IX) for SDM54, SDM61 and SDM62 are presented in Table 2 (contralateral projection) and Table 3 (ipsilateral projection). Below we report the data representing the mean of all 3 cases investigated using stereology. Notably, the general distribution patterns of bouton labeling were strikingly similar across all three cases. Individual differences between the cases can be identified in Table 2 (contralateral projection) and Table 3 (ipsilateral projection).

In terms of laterality, the heaviest amount of terminal bouton labeling was by far found in the contralateral spinal gray with few labeled terminals occurring ipsilaterally (Figs. 6-8). For example, after calculating the total mean of labeled boutons for all three M1 cases (203,812 total boutons), $98 \%$ of the total number of terminal boutons were located contralaterally $(199,214)$ while only $2 \%$ were located ipsilaterally $(4,598)$ (Tables 2, 3; Fig. 9A). Below we present our findings in order of highest to lowest labeling density for each major spinal region (Fig. 9), a breakdown of labeling within the various subsectors of laminae VII and IX (Fig. 10) and a segmental analysis of our findings (Fig. 11).

\section{Contralateral M1 Projection}

Overall Laminar Organization of Terminal Labeling-With respect to the entire contralateral projection, $80 \%$ of the total number of contralateral terminal boutons was found in the intermediate zone (laminae V, VI, VII, VIII and the RMB) (Fig. 9B; Table 2). A moderate amount of labeling (18\%) was found in the ventral horn of the spinal gray matter (lamina IX). In contrast, few terminals, (slightly less than 1\%), were found in the dorsal horn (lamina I-IV) and a few terminals were found in the contralateral half of lamina $\mathrm{X}$ (nearly $1 \%)$.

Intermediate Zone Labeling-Within the intermediate zone, the highest percentage of labeled terminal boutons was located in lamina VII (59\%) (Fig. 9B). Within lamina VII, the highest number of boutons was consistently located in three subsectors which included the ventrolateral (33\%), dorsolateral (29\%) and dorsomedial (26\%) divisions (Fig. 10A). In contrast, fewer total boutons were localized in the ventromedial (8\%) and ventral (4\%) subdivisions (Fig. 9B). A moderate and dispersed distribution of labeled terminals was found in lamina VI (14\%) (Fig. 9B) which constituted the third densest contralateral projection detected. Within lamina VI, labeling was slightly more pronounced in the medial region $(8 \%)$ than the lateral region (6\%) (Table 2). Fewer labeled boutons were found in the 
RMB (3\%) (Table 2, Fig. 9B). Similarly, approximately 3\% of the contralateral projection occurred in lamina V, with only $1 \%$ located in lamina VIII (Fig. 9B). Thus, by far the majority of labeling resided in lamina VII of the intermediate zone with the highest distribution observed in the ventrolateral, dorsolateral, and dorsomedial sectors.

Ventral Horn Labeling-In the ventral horn of the spinal gray matter, lamina IX contained on average 18\% of the total number of contralateral labeled boutons (Table 2; Fig. 9B). Within lamina IX, the heaviest labeling was distributed in the dorsomedial and dorsolateral quadrants (Fig. 10B). For example, when considering the total estimated number of contralateral lamina IX boutons in the cervical enlargement, a robust distribution of $40 \%$ was located dorsomedially with a comparable $38 \%$ located dorsolaterally. In contrast only $13 \%$ were located ventromedially and only $9 \%$ ventrolaterally.

Dorsal Horn Labeling - In the dorsal horn, bouton labeling was sparsely distributed in laminae I-III and IV (less than 1\% of the total contralateral labeled boutons) (Table 2; Fig. 9B, see laminae I-IV). In all 3 cases, labeling was found to be more pronounced in the medial part of lamina IV (Table 2). In 2 of the 3 cases studied (SDM54 and SDM61), labeling was exclusively found in the medial aspect of laminae I-III.

Segmental Organization-In the rostrocaudal dimension, a strong projection ended in C5 but the largest percentage of total bouton labeling occurred at spinal level C6 followed by a gradual decline in total number from $\mathrm{C} 7$ to T1 (Fig. 11A). For example, when considering the total number of boutons estimated for each segmental level, 23\% of labeled boutons occurred at $\mathrm{C} 5,28 \%$ at $\mathrm{C} 6,23 \%$ at $\mathrm{C} 7,18 \%$ at $\mathrm{C} 8$ and $8 \%$ was found at $\mathrm{T} 1$. There was one notable exception to this trend, in case SDM62 bouton numbers at $\mathrm{C} 8 \mathrm{did}$ not decline, but remained elevated to be similar in number (24\%) to that found at C6 (24\%) (Fig. 11A).

A more detailed level of investigation was carried out on segmental labeling in lamina VII and IX since these laminae received the strongest corticospinal input. Total bouton labeling within laminae VII showed a similar pattern as described in our overall analysis of contralateral labeling with a strong projection to $\mathrm{C} 5$, followed by a peak number of labeled boutons occurring at $\mathrm{C} 6$, then a gradual decline in total number of labeled boutons (mean numbers of labeled boutons: C5 - 29,470; C6 - 33,660; C7 - 27,731; C8 - 20,075; T1 7,456) (Fig. 11C, note black bars; Fig. 12B). In terms of labeling within the various subsectors of lamina VII, a pattern identical to our overall analysis of labeling in lamina VII was discovered. For example, the densest labeling occurred within 3 subsectors at all segmental levels, regardless of any increase or decrease in total number of labeled boutons. These included the dorsomedial, dorsolateral and ventrolateral subsectors (Fig. 11C). Interestingly, our findings demonstrated a relatively similar distribution of labeling across these three subsectors at levels C5-C6, followed by a slightly dominate presence of labeling in the ventrolateral sector at levels C6-T1 (Fig. 12B) despite the progressive overall decrease in labeling caudally. Our segmental analysis of terminal labeling in lamina IX was of significant interest in that it revealed a trend of increased terminal labeling in the rostrocaudal dimension which was opposite to the progressively decreased labeling found in lamina VII. Specifically, our investigation demonstrated that the total number of labeled boutons gradually increased from $\mathrm{C} 5$ to $\mathrm{C} 8$ in lamina IX with a slight decline at T1 (mean number of labeled boutons: C5 - 4,737; C6 - 6,078; C7 - 7,516; C8 - 10,141; T1 - 7,048) (Fig. 11E, note black bars; Fig. 12B). Another interesting pattern was revealed with respect to the spatial distribution of labeling found within lamina IX at each segment. At spinal segments C5, C6 and C7, terminal labeling was concentrated in the dorsolateral and dorsomedial quadrants with considerably less labeling in the ventral quadrants (Fig. 11E; Fig. 12B). At C8 labeling continued to be prominent in the dorsal sectors but there was a 
notable increase in the relative number of labeled boutons in both ventral quadrants. At T1 this trend continued as labeling became more uniformly dispersed amongst the quadrants. Specifically, the dorsal quadrants received only slightly more terminal corticospinal input than did the ventral quadrants at T1 (Fig. 11E; Fig. 12B).

Ipsilateral M1 Projection-Our stereological analysis demonstrated that the ipsilateral projection from M1 was primarily confined to the intermediate zone and midline region (lamina X) with very few terminals occurring in the ventral horn (Table 3). No labeling was found in the dorsal horn.

With respect to the total number of labeled boutons estimated for the ipsilateral projection, the highest amount of labeled profiles was found in lamina VII (67\%) (Fig. 9C) with 14\% of labeled boutons located in lamina VIII and $14 \%$ occurring in the ipsilateral half of lamina X. Other regions found to contain sparse terminal labeling included laminae IX (3\%), VI (2\%) and V (1\%) (Fig. 9C).

Within lamina VII our analysis revealed that in all 3 cases, labeling was most prominent in the dorsomedial (44\%) and ventromedial (37\%) sectors (Fig. 10C). Much less terminal labeling was found in the ventrolateral (10\%) and dorsolateral (6\%) regions, with very few terminals localized in the ventral region $(3 \%)$.

As noted above, lamina IX labeling was sparse (representing 3\% of the total number of ipsilateral labeled boutons) and was found using our stereological probe in only one experimental case (SDM62) (Fig. 10D). In this case, labeling was located primarily within the dorsomedial and ventromedial quadrants with a few boutons noted in the ventrolateral region.

Our segmental analysis of the ipsilateral projection revealed a relatively high number of boutons in all cases at $\mathrm{C} 5$, very low numbers at $\mathrm{T} 1$ and a more variable pattern of labeling in between (C6 to $\mathrm{C} 8$ ) that was generally characterized by a gradual decrease in overall terminal labeling (Fig. 11B). In fact, when the findings from all 3 cases were averaged, there was a picture of stepwise decreases in labeled boutons from C5 to C6/7 and then to C8/T1, but no single case actually demonstrated this. For example, in case SDM54, the highest estimated number of boutons was found at C6, in case SDM61 labeling at C8 was slightly higher at than C7, and in case SDM62 a very large number of labeled boutons were estimated to occur at level C7. Our segmental analysis of labeling within the various subsectors of lamina VII was more consistent indicating most labeling occurred in the dorsomedial and ventromedial subsectors at $\mathrm{C} 5$ to $\mathrm{C} 8$ (Fig. 11D) except at C7 where labeling was highest in the dorsomedial and ventrolateral subsectors. At T1, labeling was found primarily in the dorsolateral region of lamina VII, adjacent to lamina IX. Lamina IX labeling was found only at levels C5 and C7 underscoring its rare occurrence in our case material (Fig. 11F). At both levels, labeling was weak, similar in intensity and located primarily in the medial region of lamina IX.

\section{DISCUSSION}

Deeply engrained in the clinical and experimental literature is the fact that the corticospinal projection (CSP) from the primary motor cortex (M1) represents a key descending pathway that plays a central role supporting the capacity to execute highly coordinated arm, hand and digit movement (Lassek, 1954; Kuypers, 1978, 1981; Davidoff, 1990; Lemon, 1993; Porter and Lemon, 1993). The current study represents the first effort to anatomically characterize the terminal distribution of this important descending pathway in the cervical enlargement of the monkey using a comprehensive stereological design in combination with high-resolution 
dextran tract tracers and intracortical microstimulation. With these applications, we were able to find that the general pattern of labeled terminal boutons, resulting after tracer injection confined to the hand/arm region of M1 in three animal experiments was strikingly consistent in terms of laterality, relative terminal labeling intensity and topographical distribution within discrete sub-regions of Rexed's laminae.

Our findings show an almost exclusively contralateral projection which principally targets lamina VII and to a lesser extent, laminae IX and VI in a highly organized manner (Figs. 6$8,12 \mathrm{~A}$ ). The projection to lamina VII was most dense in the dorsomedial, dorsolateral and ventrolateral quadrants at all cervical levels investigated (Fig. 12B). We also found a gradual decrease in the number of labeled terminals in lamina VII progressing from spinal level C5 to spinal level T1. This pattern was accompanied by an increase in the number of lamina IX terminals from $\mathrm{C} 5$ to $\mathrm{C} 8$, with a slight decline of labeled terminals at the $\mathrm{T} 1$ level compared to C8 (Fig. 12B). Additionally, we found a progressive shift in terminal labeling within the various quadrants of lamina IX from C5 to T1 that was portrayed as initially being restricted to the dorsal region of lamina IX at rostral and mid-levels of the cervical enlargement (C5, C6 and C7) followed by a pattern that was characterized as being more diffusely distributed at inferior segmental levels (C8 and TI). The CSP projection from M1 to laminae VI, V and $\mathrm{IV}$, was found to be more prominent medially, suggesting a common region of overlap with corticospinal input from somatosensory areas $3 \mathrm{~b}, 1$ and 2 of the adjacent postcentral gyrus (Coulter and Jones, 1977; Ralston and Ralston, 1985).

\section{Laterality of the M1 Corticospinal Projection}

As mentioned in the Introduction, a wide range of observations characterizing the proportion of contralateral versus ipsilateral projections from the M1 arm region have been reported. Some of these studies have been limited by quantitative methodology such as the optical density method and other studies have focused on estimating axon number in descending white matter pathways. Our stereological analysis of discretely labeled terminal boutons throughout the spinal gray matter and reticulated marginal border demonstrated a strikingly dominant contralateral projection that represented approximately $98 \%$ of the total number of estimated boutons in all three experimental cases with a mere $2 \%$ of this terminal projection located ipsilaterally (Fig. 9A). The scarcity of ipsilateral bouton labeling in our study is reinforced by the recent findings showing that after injection of BDA into M1, some labeled fibers descending in the ipsilateral lateral corticospinal tract enter the ipsilateral spinal gray only to pass through this region, cross the midline, and then enter the contralateral gray matter (Rosenzweig et al., 2009; Yoshino-Saito et al., 2010). Functionally, our finding of very limited numbers of ipsilateral terminal boutons is supported by the lack of physiologically evoked ipsilateral movements from the precentral region of M1 (area 4) in the rhesus macaque following surface stimulation (Bucy, 1933; Bucy and Fulton, 1933) and intracortical microstimulation (Soteropoulos et al., 2011; Zaaimi et al., 2012). Also suggestive of a small number of ipsilateral M1 projections is the limited role of an ipsilateral corticospinal contribution to motor recovery in the non-human primate that was elegantly documented by Zaaimi and co-workers (Zaaimi et al., 2012).

\section{Terminal Projection of M1 to Contralateral Lamina IX}

As previously emphasized, a hallmark feature of the M1CSP is its direct contralateral projection to motoneurons located within lamina IX. In our study, the contralateral M1 projection to lamina IX comprised the second strongest projection in agreement with Dum and Strick (1996) as it averaged approximately $18 \%$ of the total contralateral projection (Fig. 9B). Our initial analysis of the total number of labeled boutons in the cervical enlargement (C5-T1) demonstrated that the projection preferentially innervates the dorsomedial and dorsolateral quadrants of lamina IX (Table 2). This finding is consistent with studies that 
have shown dense terminal labeling in the dorsomedial (Kuypers and Brinkman, 1970; Maier et al., 2002; Rosenzweig et al., 2009) and dorsolateral (Kuypers and Brinkman, 1970; Ralston and Ralston, 1985; Dum and Strick, 1996; Rouiller et al., 1996; Amand et al., 1997; Maier et al., 2002; Yoshino-Saito et al., 2010) subregions following ablation, or tract tracer injection involving the upper extremity representation of M1.

In our segmental analysis of lamina IX labeling, we found that the average number of labeled terminals was highest at levels C7, C8 and T1 compared to C5 and C6 (Fig. 11E) which is very similar to previous observations characterizing the M1 CSP to lamina IX following tracer injections involving the anterior bank of the central sulcus (Dum and Strick, 1996; Maier et al., 2002; Yoshino-Saito et al., 2010, see their Fig. 7, monkeys 1 and 2). The stepwise increase found in our study closely parallels the WGA-HRP observations (Dum and Strick, 1996; Armand et al., 1997; Maier et al., 2002) with one exception. All of these studies found an increase in lamina IX labeling from segmental level C8 to T1, whereas we found a slight drop in labeled terminals from C8 to T1 (Fig. 11E). This difference could be due to axonal transport issues which will be discussed, or the densitometric method applied in these previous studies. With respect to the method of terminal quantification, we found a more homogenous distribution of terminal labeling within the four quadrants at T1 than at C8. For this to occur, affiliated labeled axons would be more widely dispersed in lamina IX at T1, possibly increasing the densitometric values. An additional aspect to be considered is the possibility of a progressive reduction of lamina IX area/volume at the T1 level compared to the $\mathrm{C} 8$ level with little change in the number of labeled boutons and axons at both levels (Fig. 5, compare the surface area of lamina IX in panels $\mathrm{G}$ and $\mathrm{H}$ to I and J; Fig. 12B, compare lamina IX at levels C8 and T1). The net result could be the appearance of a much denser distribution of labeling at $\mathrm{T} 1$. Other differences could be related to interpreting the relative boundary between $\mathrm{C} 8$ and $\mathrm{T} 1$. Interestingly, a slight drop in lamina IX labeling from C8 to T1 was reported by Yoshino-Saito and co-workers in their study (Yoshino-Saito, et al., 2010 see their Fig. 7, case Mo 2). Despite these differences, this work collectively demonstrates the M1 hand/arm region preferentially innervates lamina IX at levels C8 and T1 which in turn, innervates muscles acting on the wrist, hand and digits (Jenny and Inukai, 1983). In terms of topography, our finding of a more widespread pattern of terminal labeling in lamina IX at C8 and T1, that gradually includes more terminals in the ventral region of lamina IX (Figs. 6, 7, 8- see levels C8 and T1; 11E), corresponds well with the observation that a great deal of lamina IX surface area is occupied by terminal labeling at these levels (Armand et al., 1997; Lemon et al., 2002; Maier et al., 2002) compared to the predominantly dorsal labeling we found in C5-C7 (Figs. 6, 7, 8 - see levels C5, C6 and C7; Figs. 11E, 12B).

When considering what is known about the musculotopic organization of monkey motoneurons in the cervical enlargement and our segmental analysis of lamina IX labeling, some functional inferences can be advanced for future physiological investigation. It is possible to speculate that the preferential projection to the dorsal quadrants at C5, C6 and C7 (Fig. 12B) may heavily influence flexor muscles acting on the shoulder and elbow since biceps brachii motoneurons are present in large numbers dorsally at these cervical levels (Jenny and Inukai, 1983). In contrast, motoneurons innervating muscles acting on the wrist and digit joints are primarily located at levels C7 - T1 with flexors generally located dorsally to functionally paired extensors (Jenny and Inukai, 1983). Thus, the more dispersed pattern of terminal innervation found in our analysis at caudal cervical levels suggest potential influence on flexors, extensors, abductors and adductors acting on the wrist and fingers. It is important to note that these inferences must be tempered by the finding that motoneurons innervating flexor and extensor muscles of the hand, wrist, elbow and shoulder partially overlap in lamina IX, gradually shift in location along the rostral-caudal axis, and display intermingled dendritic fields (Jenny and Inukai, 1983; Chiken et al., 2001). Furthermore, macaque monkey motoneurons give rise to dendritic processes that extend beyond the 
classically assigned anatomical boundary of lamina IX (Lawrence et al., 1985; Rouiller et al., 1996) as demonstrated in other species (for review see Kuypers, 1981).

\section{Terminal Projection to the Contralateral Intermediate Zone}

Gray matter forming contralateral lamina VII was the primary target for the M1 CSP (Fig. 9B) which is consistent with previous reports that have quantitatively evaluated the laminar distribution of labeling in the monkey cervical enlargement (Dum and Strick, 1996;

Yoshino-Saito et al. 2010). Given that considerable uncertainty continues to exist regarding the topographical distribution of this projection, we attempted to quantify the terminal distribution in five distinct subsectors of lamina VII using stereology. We found in all three experimental cases that the dorsomedial, dorsolateral and ventrolateral subsectors consistently contained large numbers of labeled terminal boutons in all five spinal segments, with the ventromedial and ventral regions containing substantially lower numbers of labeled boutons (Figs. 10A, 11C, 12B). Thus, our findings verify the numerous accounts of a strong M1 hand/arm area projection to the dorsolateral part of lamina VII (Kuypers and Brinkman, 1970; Kucera and Wiesendanger, 1985; Ralston and Ralston, 1985; Dum and Strick, 1996; Armand et al., 1994, 1997; Maier et al., 2002; Yoshino-Saito et al., 2010) as well as dense labeling noted in the ventrolateral (Kuypers and Brinkman, 1970; Dum and Strick, 1996; Armand et al., 1994, 1997; Maier et al., 2002) and dorsomedial (Kuypers and Brinkman, 1970; Armand et al., 1997) regions of lamina VII. We did not find a strong contralateral projection to the ventromedial region of lamina VII (and adjacent VIII) as previously reported in the monkey model (Dum and Strick, 1996). Thus, it is possible that the ventromedial region of lamina VII contains high numbers of labeled fibers of passage and possibly, M1 labeled axons crossing the midline that have been noted in several studies (Sherrington, 1889; Liu and Chambers, 1964; Ralston and Ralston, 1985; Leichnetz, 1986; Galea and Darian-Smith, 1997; Rosenzweig et al., 2009; Yoshino-Saito et al., 2010).

Although many crossing fibers noted in the Rosenzweig et al., report (2009) are likely to involve bilateral influences from the injection involving the leg and proximal arm areas. It is also possible that the dense labeling reported for the ventromedial region of lamina VII (and adjacent lamina VIII) by Dum and Strick (1996) could be related to potential injection site involvement (their Case R4) of the shoulder representation of M1, since the shoulder region preferentially projects to the contralateral ventromedial part of lamina VII and adjacent part of lamina VIII (Kuypers and Brinkman, 1970, see their Fig. 1, case 18). In agreement with our findings, is the observation of Maier and colleagues (Maier et al., 2002) who found a strong contralateral projection from the physiologically defined M1 hand/arm representation to the dorsolateral part of the intermediate zone, with comparatively lighter labeling occurring in the ventromedial part of lamina VII and adjoining part of lamina VIII. Furthermore, if the arm area of the supplementary motor cortex (M2 or SMC) in the opposite hemisphere was not injected with HRP in this study (Maier et al., 2002, see Fig. 2, monkey case CS8) we would predict that labeling in the ventromedial part of lamina VII and adjacent lamina VIII would have been even lighter than reported by Maier and co-workers. This would be based on our previous work (McNeal et al., 2010) showing that the primary ipsilateral projection from M2 targets lamina VIII and the medial part of lamina VII, and thus, may have added to the overall density of the M1 projection shown terminating in this spinal region in their case CS8. Other work (Armand et al., 1997) would also suggest that injection site involvement on the gyral surface of the M1 hand/arm region, in addition to the dorsally located shoulder region, may account for enhanced bilateral labeling in lamina VIII. Finally, our finding of a weak projection to contralateral lamina VIII would be consistent with several accounts of this projection from the hand/arm region of M1 (Rouiller et al., 1996; Maier et al., 2002; Yoshino-Saito et al., 2010). 
Our segmental analysis of the lamina VII projection clearly indicated that the occurrence of terminal bouton labeling within lamina VII gradually decreases, in a stepwise manner from C5 to T1 (Figs. 9C, 12B). This finding is in opposition to the observations of Yoshino-Saito and colleagues (2010) who reported a gradual increase in BDA labeled terminals from C5 to C8 following injection of BDA into the M1 hand/arm region (their Fig.7, case Mo 1-2). Such differences could be due to the diverse methodological applications used to estimate bouton number. In the present study, we applied an unbiased stereological analysis design with the optical fractionator probe that included an evaluation of every other tissue section within each segmental interval (totaling 5 to 6 horizontal tissue sections for each segmental level). Our counting frame $(109.2 / 71.4 \mu \mathrm{m})$ and $\mathrm{X} / \mathrm{Y}$ grid placement $(125.3 / 241.9 \mu \mathrm{m})$ applications permitted us to examine bouton labeling in 300 to 400 gray matter sampling sites for each individual tissue section (150-200 counting frame sites per gray matter side). In contrast, stereology was not used in the Yoshino-Saito report (Yoshino- Saito et al., 2010). These differences could also be due to the different post-injection survival periods applied which will be discussed under "Methodological Considerations".

We found a sizeable projection to lamina VI representing the third strongest contralateral projection (14\% of the total contralateral projection) corresponding with previous observations (Dum and Strick, 1996; Maier et al., 2002). Our finding in all three cases investigated, further indicates that this projection primarily targets the medial region of lamina VI (Table 2) in agreement with Dum and Strick (1996). Finally, with respect to intermediate zone labeling we localized a weaker projection to lamina $\mathrm{V}$ compared to lamina VI in accord with others (Dum and Strick, 1996; Yoshino- Saito et al., 2010). In two of our cases, labeling in lamina $\mathrm{V}$ was concentrated more medially and in one case we found an equally dispersed projection amongst the medial and lateral subsectors (Table 2).

Intermediate zone labeling provides information on the potential synaptic connections of M1 neurons onto interneurons (and dendrites from somas located outside of this zone) that are likely involved in spinal circuits that transform motor cortex output into muscle-level commands. Although some motor cortex output is distributed directly onto spinal motoneurons, it is clear that the majority of its output terminates in the intermediate zone where neuronal recordings in non-human primates have demonstrated their involvement in coordinate transformations for control of wrist muscles when performing isometric tasks (Fetz et al. 2002, Yanai et al. 2007, Shalit et al. 2012). Yanai and colleagues (2007) also suggested that spinal interneurons transform variable/evolving cortical motor commands into consistent commands for activating spinal motoneurons to produce isometric wrist torques. However, it is commonly assumed that direct activation of spinal motoneurons is especially important for control of digit musculature for independent finger movements and precision grip (for review see Cheney et al., 1991; Lemon, 1993, 2008; Porter and Lemon, 1993; Lemon et al., 2004; Lemon and Griffiths, 2005; Schieber, 2007). Our findings of a reduced number of corticospinal terminal boutons in intermediate zone laminae of $\mathrm{C} 8$ and T1, coupled with increased boutons in lamina IX are supportive of this hypothesis because the motoneurons of intrinsic finger muscles are located at these segmental levels. It is possible that transformation of cortical motor commands by spinal interneurons in intermediate laminae for activation of spinal motoneurons is most important for control of shoulder, elbow and wrist torque or motion while cortical commands for finger movement need less transformation (hence, the smaller number of boutons in intermediate zones in C8, T1 near spinal motoneuron pools for these muscles). Although the transformation to movement is considerably more complex than the transformation to isometric torque studied in previous work (e.g., Fetz et al., 2002; Yanai et al., 2007) because of interaction torques arising from motion of multiple segments, which are significant even for finger motions (Darling and Cole 1990). Thus, although there are fewer M1 output connections onto intermediate laminae neurons at the segmental levels containing finger muscle motoneurons 
(C8 and T1) it is possible that those connections are important for production of coordinated finger movements. Moreover, recent work has suggested that premotor interneurons located in the intermediate zone of C5-C7 in addition to C8-T1 are involved in control of grasping by coordinating multiple finger muscles in primates (Takei and Seki 2010). Propriospinal neurons at $\mathrm{C} 3$ and $\mathrm{C} 4$ segmental levels may also mediate cortical motor commands for precision grip even in the absence of direct corticospinal connections on spinal motoneurons of finger muscles (Sasaki et al. 2004), probably through direct intraspinal projections onto motoneurons of C6-T1 (Isa et al., 2006; 2007; Alstermark et al., 2011).

\section{Terminal Projection to the Contralateral Dorsal Horn}

The dorsal horn of the spinal cord is classically known to participate in sensory related mechanisms (for review see Morris et al., 2004; Basbaum et al., 2009; Todd, 2010). In our study, we found a weak projection to this region (Fig. 9B; Table 2) as noted by others (Ralston and Ralston, 1985; Dum and Strick, 1996; Armand et al., 1997; Maier et al., 2002; Yoshino-Saito et al., 2010). Furthermore, our findings suggest that this projection is primarily directed to the medial region of the dorsal horn. Consideration of the more medially distributed projection from M1 to lamina VI, V and IV (and medial part of laminae I-III in 2 of the 3 cases), it appears as if this component of the M1 projection selectively overlaps with the more discrete corticospinal projection from somatosensory areas $3 \mathrm{~b}, 1$ and 2 of the adjacent postcentral gyrus (Coulter and Jones, 1977; Ralston and Ralston, 1985). Thus, the general principle of corticofugal organization stating that reciprocally interconnected cortical areas project in part, to similar (overlapping) regions of striatum (Yeterian and Van Hoesen, 1978), may apply to the corticospinal projection. Particularly when considering the overlap of the CSP from M1 and supplementary motor cortex (Dum and Strick, 1996; Rouiller et al., 1996; Lemon et al., 2002; Maier et al., 2002) as discussed below.

\section{Terminal Projection to the Ipsilateral Spinal Gray Matter}

We found a weak ipsilateral projection which was in part, addressed under the previous discussion on laterality. With respect to laminar labeling, we found this projection to preferentially target ipsilateral lamina VII, VIII and X. Our findings show a stronger ipsilateral projection to lamina VII than VIII (Fig. 9C) in agreement with Dum and Strick (1996) and we also identified a relatively strong ipsilateral projection to lamina X (Fig. 9C). Our results demonstrate a very weak ipsilateral projection to laminae IX, VI and V in agreement with previous reports (Dum and Strick, 1996; Yoshino-Sato, et al., 2010). Furthermore, the lack of a significant ipsilateral corticospinal projection to lamina IX support the physiological findings of Soteropoulos and co-workers (2011) who found little evidence for direct ipsilateral corticomotoneuronal projections in the monkey. Finally, our overall analysis (Fig. 10C) indicated that the medial region of lamina VII is the primary recipient of ipsilateral M1 corticospinal output. This pattern was also found in our segmental analysis (Fig. 11D) with the exception of level T1, which had intermediate zone labeling primarily in the ventrolateral subsector of lamina VII, in close proximity to adjacent lamina IX. Functionally, the ipsilateral projection to lamina VIII and the medial part of lamina VII indicates some potential influence over shoulder and postural movements since interneurons in these regions project to proximal and axial muscle motoneurons (Holstege, 1988; Mouton, and Holstege, 1994). These connections may additionally subserve the apparent successful performance of visually guided arm movements by the limb ipsilateral to the nasal half of the visual field of one eye observed in split-brain monkeys by Kuypers and colleagues (Brinkman and Kuypers, 1972, 1973; Haaxma and Kuypers, 1975). The lack of ipsilateral M1 connections into lamina IX may explain the poor visually guided grasping by the hand and digits ipsilateral to the nasal half of the visual field in these split-brain monkeys. 


\section{Comparisons with the Supplementary Motor Cortex Corticospinal Projection}

A limited number of neuroanatomical studies (Rouiller et al., 1996; Dum and Strick, 1996; Lemon et al., 2002; Maier et al., 2002) have concurrently examined features of contralateral CSP from M1 and the supplementary motor cortex (M2, MII, SMC, SMA, SSMC or SMAProper) to identify shared, as well as distinguishing features of these two major frontal corticofugal projection systems. In line with these studies, our findings clearly show that the M1 projection ends in parts of the contralateral intermediate zone (lamina V, VI, VII and VIII), and ventral horn (lamina IX) also receiving M2 input (Rouiller et al., 1996; Dum and Strick, 1996; Lemon et al., 2002; Maier et al., 2002; McNeal et al., 2010). Considering our previous report on the terminal distribution of the M2 projection (McNeal et al., 2010), which employed a similar experimental tract tracing design as the present study, our data suggest some additional similarities and differences amongst the M1 and M2 projection to the intermediate zone. Both motor areas appear to project more densely to the lateral part of lamina VII although in our previous study (McNeal et al., 2010) we did not subdivide lamina VII into 5 compartments as we did for the present work. In contrast, on average the projection to lamina VI and V was densest medially for the M1 CSP and laterally for the M2 CSP.

Our current study and the McNeal report (McNeal et al., 2010) support previous work (Dum and Strick, 1996; Rouiller et al., 1996; Lemon et al., 2002; Maier et al., 2002) showing that the contralateral projection from both M1 and M2 preferentially targets the dorsal part of lamina IX (Fig. 9B; McNeal et al., 2010, see their Table 4). However, as we found in our segmental analysis, and as noted by others (Dum and Strick, 1996; Maier et al., 2002), this pattern included more labeling ventrally at levels C8 and T1 for the M1 CSP (Fig., 11E) which may not be a common characteristic for the M2 CSP at C8 and T1 (Maier et al., 2002). In agreement with previous work we found a 3 -fold higher total number of contralateral lamina IX terminals from M1 (36,464: average of 3 cases) compared to M2 (11,277; average of 4 cases) coinciding with physiological observations demonstrating that M1 monosynaptic connections on motoneurons are far greater and exert stronger excitatory effects on upper extremity musculature than M2 (Lemon et al., 2002; Maier et al., 2002; Boudrias et al., 2006, 2010a, 2010b). Interestingly, we found a much wider range of estimated lamina IX terminals amongst the four animals in our M2 case analysis (McNeal et al., 2010, see Table 2) than amongst our three M1 cases (Table 2) suggesting that in addition to the substantially weaker M2 input to lamina IX, this terminal projection may be much more variable from animal to animal, than the M1 motoneuron projection.

Findings from our laboratory provide strong support for previous conclusions that have shown the overall M1 upper extremity CSP is much denser than the M2 CSP (Dum and Strick, 1996; Lemon et al., 2002; Maier et al., 2002). In the current report, we found an average number of contralateral M1 terminal boutons to be 199,214 (average of 3 animals) compared to an estimated 75,853 contralateral M2 boutons (average of 4 animals) observed in our previous report (McNeal et al., 2010). It is important to note that in both studies, we employed a compatible dextran tracer design that included injecting the identical total tracer volume $(1.2 \mu \mathrm{L})$ discharged into three equally spaced penetration points $(0.4 \mu \mathrm{L}$ per site) and directly into a central region of the physiologically defined arm area of M1 and M2 in each experimental case. This difference in total bouton number compliments the 3-4 fold preponderance of M1 corticospinal projection neurons found in M1 versus M2 after retrograde tracer injection into the spinal cord (He et al., 1993, 1995). It is important to state that our estimated number of M1 terminals $(199,214)$ is only representative of a small portion of the M1 forelimb region whereas our estimated number of M2 terminals is probably representative of a larger proportion of the $\mathrm{M} 2$ forelimb region, particularly when considering our analysis demonstrated similar values for the injection site halos in both studies (and thus similar tract tracer spread) (Table 1, see injection site halo; Table 1, see 
control cases, of McNeal et al., 2010). Indeed, the entire supplementary motor cortex (face, forelimb, hind limb and trunk representations) is roughly the same size as the M1 forelimb representation alone (Wise, 1996). This is notable in Figure 1 showing that a large portion of the hand/arm region of M1 located dorsal, ventral and rostral to each injection site was not involved in the injection site core or halo zones. Indeed, this was intended to ensure the entire volume of tract tracer was placed solely within the neuronal uptake region of the M1 hand/arm representation while simultaneously avoiding tracer uptake from the adjacent shoulder (dorsally) and face (ventrally) representations of M1. The latter scenario could have negatively affected the total estimated bouton numbers calculated for any given case by reducing the availability of tracer to effectively fill hand/arm terminal boutons due to the forfeiture of tracer to the M1 shoulder and face axon transport systems. In addition, involvement of the M1 shoulder representation may have altered the overall terminal projection pattern as discussed.

Bearing in mind that the density of corticospinal neurons per unit area is only slightly greater in M1 than M2 (Dum and Strick, 1991; Luppino et al., 1994; He et al., 1995; see Wise, 1996 for review), and the core volumes of our M1 injection sites were much smaller than our M2 injection sites (with similar halo volumes) (Table 1; see Table 1, control cases, of McNeal et al., 2010) it is possible to speculate from our findings that there are significant differences in the total number of corticospinal terminals issued from a similar number corticospinal neurons originating from these motor areas, with neurons from M1 giving rise to a much more extensive network of terminal boutons.

Previous studies have not examined the relationships of the ipsilateral projection from M1 and M2. In terms of laterality, there appears to be a significant difference between these projection systems. In the current study we found the M1 projection to be almost exclusively contralateral (98\% contralateral and 2\% ipsilateral, see Tables 2, 3; Fig. 9A). In profound contrast, in our previous study (McNeal et al., 2010) we found the M2 contralateral projection to represent $81 \%$ of the total projection with a substantial $19 \%$ of the projection located ipsilaterally (McNeal et al., 2010, see their Tables 2 and 3). Thus, the supplementary cortex CSP appears to be in a much stronger position connection wise, to mediate bilateral spinal mechanisms. Much like the contralateral projection, the topography of the M1 and M2 ipsilateral projections appear to be very similar in that both motor cortices preferentially innervate laminae VII and VIII. In addition, both motor areas project preferentially to the medial region of lamina VII (M1 $-81 \%$ of the ipsilateral projection ended medially and $12 \%$ terminated laterally, see Figs. 10C, 11D; M2 - 53\% of the ipsilateral projection ended medially and $6 \%$ terminated laterally, see Table 3 of McNeal et al., 2010). In addition to the large differences in laterality, our observations indicate that the projection from M2 is much denser to ipsilateral lamina VIII than is the projection from M1. Finally, the significant ipsilateral projection to lamina X may be unique to the M1 CSP.

\section{Kuypers "Lateral Motor System" Revisited}

In addition to attempting to gain new insight on the topographical distribution of the M1 corticospinal projection to lamina VII, our experimental plan was in part, designed to test Kuypers conceptualization of a "lateral motor system" (Lawrence and Kuypers, 1968a; Kuypers, 1981; 1982; for contemporary reviews, see Lemon, 2008; Lemon et al., 2012) using modern dextran tracers, since several recent studies have not found evidence to support his theory (Bortoff and Strick, 1993; Dum and Strick, 1996). Briefly, Kuypers "lateral motor system" stresses the importance of contralateral descending projection systems affecting distal limb movements. In contrast, axial and proximal limb movements are largely regulated by a "medial motor system" (Lawrence and Kuypers, 1968b; Kuypers, 1981, 1982). His concept of both lateral and medial motor systems was further strengthened 
by observations of bulbospinal projection patterns and the longitudinal organization of propriospinal interconnections found in the cat model (for review see Kuypers et al., 1982).

With respect to the lateral motor system concept and the corticospinal projection, this theory was heavily grounded by Kuypers' observations demonstrating that the caudal hand region of precentral (primary) motor cortex (M1) preferentially targets the contralateral dorsomedial, dorsolateral and ventrolateral parts of the intermediate zone in addition to a significant projection to the ventral horn (lamina IX) at the spinal enlargements (Fig. 4B). The findings from our study provide strong support for Kuypers "lateral motor system" model from the standpoint of the M1 hand/arm projection to the cervical enlargement of the spinal cord. Indeed, at all spinal levels investigated, there was prominent labeling in the dorsomedial, dorsolateral and ventrolateral regions of lamina VII (Figs. 10A, 11C) that was spatially continuous with heavy labeling that spread ventrally into adjacent lamina IX (Figs. 10B, 11E, 12B).

In contrast, as mentioned above, Kuypers "medial motor system" notion is related to central nervous system governance of proximal and axial limb movements. With respect to the corticospinal connection, he viewed the M1 shoulder area, rostral primary motor cortex (on the gyral surface) and dorsolateral premotor cortex projection to be part of this system since these corticospinal connections "carry representations of axial and proximal movements" and preferentially target the contralateral ventromedial region of the intermediate zone and adjacent lamina VIII (Fig. 4B) (Kuypers, 1981; 1982). These projections were also noted to be more bilateral in nature than the descending projections affiliated with the lateral motor system principle that would in turn support bilateral axial and proximal limb movements. Our findings lend complementary support for this viewpoint since significantly lower numbers of contralateral labeled boutons were found in the ventromedial and ventral subsectors of lamina VII (Figs. 10A, 11C) that was accompanied by an extremely sparse allocation of labeling in adjacent contralateral lamina VIII (Figs. 9B). From an ipsilateral standpoint, we found an extremely weak projection to lamina VIII and the ventromedial subsector of lamina VII from the hand/arm region of M1 which would by definition, fit nicely into this overall concept.

\section{Methodological Considerations}

In the current study, we employed two different dextran tracers (BDA and LYD) to accomplish our goal of estimating the terminal corticospinal projection from the hand/arm region of M1 in conjunction with stereology. Therefore, it is possible that the use of different tracers with potentially different transport characteristics may have, in itself, resulted in quite different projection estimates and possibly different projection patterns. Nonetheless, we found a remarkably similar number of estimated boutons in all three cases investigated with stereology (Tables 2, 3). Strengthening this correlation was the highly reproducible projection pattern noted in all three cases in terms of percent total of labeled boutons found within each Rexed's lamina including the various subsectors (Figs. 9B, $10 \mathrm{~A}, \mathrm{C})$. These observations, coupled with a relatively low amount of variance strongly indicate a repeatable measure of neuronal projection. Thus, in the monkey, BDA and LYD seem to be compatible dextran tracers for examining the terminal distribution of long corticofugal projection systems.

The interpretation of the precise location of the effective injection site has been a fundamental challenge in tract tracing methodology (Mesulam, 1982). As we have reviewed (McNeal et al., 2010), following tract tracer injection, extracellular dissipation occurs forming an indiscriminate halo around a dark core of reaction product characterized by a dense histochemical precipitate (Fig. 2). The injection site core is conventionally considered part of the "effective injection site" implying that tract tracer uptake and axon transport of 
the tracer actively occurs at this location (Edwards and Hendrickson 1981; Warr et al., 1981; Mesulam, 1982; Condé 1987). The uncertainty of the effective uptake site lies in the interpretation of the injection site halo, where there is a gradual decline in the density of labeled perikarya, particularly toward the periphery of the injection site halo (Fig. 2). That portion of the injection site halo closely surrounding the core is conventionally considered part of the "effective uptake zone" (Edwards and Hendrickson 1981; Warr et al., 1981; Mesulam, 1982; Condé 1987). However, beyond the inner part of the injection site halo, uptake characteristics are uncertain (i.e., in the outer halo region). In addition, very little is known about the effective uptake component for the injection site halo for dextran tracers in the monkey model, particularly for LYD which tends to dissipate more widely than BDA (for comparative examples see Morecraft 2007a, Fig. 2; Morecraft et al., 2007b, Fig. 8; McNeal et al., 2010, Fig. 1). In the present study, some insight into the role of the halo with respect to the effective transport region is afforded since we injected both BDA and LYD in the same manner and in the same cortical region. Both tracers were also transported over a 33 day period, processed in an equivalent manner to avoid inter-experimental fluctuations and analyzed using an unbiased stereolological approach. As mentioned, we found a remarkable similarity in total number of estimated labeled boutons in the BDA experiment compared to both LYD experiments (Table 2). It is also clear, that our estimated halo volume for our BDA case was much smaller than the halos of both LYD cases (Table 1). It thus appears that the effective uptake zone of the LYD tracer may be limited to the innermost region of the halo area, that closely surrounds the core as suggested for HRP (Mesulam, 1982). From another technical standpoint, it has been shown that damaged axons in subcortical white matter pathways by the syringe tip can be a source of unintended tract tracer uptake (Mesulam, 1982; Schofield, 1990; Reiner and Honig, 2006). In the current study, white matter damage from the injection procedure did not involve any longitudinal pathways or descending pathways from adjacent motor areas (Morecraft et al., 2002; Schmahmann and Pandya 2006) as verified in our histological analysis.

In the literature, there is a wide range of survival periods between dextran tract tracer injection into the monkey forelimb region of M1, and tissue fixation ranging from 21 days (Rouiller et al., 1996) to 104 days (Yoshino-Saito et al., 2010). Since adequate compound transport over a given period of time is necessary to properly label an axon system of interest, and this process is dependent on the specific transport rate that the compound is moved down the axon over time (approximately $3 \mathrm{~mm}$ day for BDA in the rodent model, Reiner et al., 1993), it is possible that our findings showing decreased bouton labeling in lamina VII progressing caudally following a 33 day survival period (Fig. 9C), versus the increased number of boutons reported by Yoshino-Saito and colleagues (Yoshino-Saito et al., 2010, see their Fig. 7), may be due to an inadequate post-injection transport survival period. However, following a 21 day survival interval Rouiller described significant anterograde transport of BDA throughout the cervical spinal cord (Rouiller et al., 1996, see their Fig. 2). Another monkey study, which injected biotinylated dextran into the cervical spinal cord (C5 to T1) and allowed for three different survival periods (21, 35 and 49 days), reported large numbers of retrogradely labeled corticospinal cells and anterogradely labeled axon terminals in thalamus and globus pallidus following all three survival periods (Brandt and Apkarian, 1992). They found excellent long range anterograde terminal filling at the 35 day interval (comparable to our 33 day survival) that was similar in quality to the 49 day interval. One important outcome in our study that would further argue for an adequate postinjection survival period was the gradual increase in terminal labeling found in lamina IX from C5 to C8 (Figs. 10E, 12B). Finally, we observed BDA and LYD labeling at spinal levels T2-T3 indicating effective tracer transport below the spinal region studied, which is similar to that reported by Yoshino-Saito et al. (2010) who after a survival period of 80-104 days noted "only a small number of axons descended caudal to the Th2" level. 
The current study attempted to quantify the M1 corticospinal projection by estimating the number of terminal-like immunoreactive particles (boutons) and we have previously discussed the nature of interpreting these particles as being representative of synaptic terminals (Morecraft et al., 2007b; McNeal et al., 2010). In the present study we have stressed the association of the locations of labeled terminal boutons with gray matter confined to each of Rexed's lamina, and defined gray matter subsectors within these laminae. It is likely that these terminals will interact with cell bodies and their dendrites confined to these regions, but some caution should be exercised in these interpretations. For instance, there are likely to be dendrites within these compartments that are affiliated with cell bodies that are located in adjacent laminae, as we have previously pointed out for cell bodies in lamina IX which give rise to dendritic extensions coursing outside of the anatomically defined boundary of lamina IX. It is likely that cells other than interneurons and propriospinal neurons reside within lamina VII, and cells other than (alpha) motoneurons reside within lamina IX. For example, neurons associated with the spinocerebellar system have been localized in lamina IX in the cat (Xu and Grant, 1988), last order interneurons receiving input from propriospinal neurons have been identified in lamina IX in the cat (Alstermark et al., 1990), and gamma motoneurons are also located in lamina IX (Bryan et al., 1972; Strick et al., 1976), although they are considered motoneurons.

\section{Summary and Conclusions}

Overall, our current understanding of the neuroanatomical organization of the corticospinal projection from the hand/arm region of M1 in the non-human primate discloses a profile of anatomical complexity and specificity that has long been suggested in the physiological and behavioral observations (Fig. 12). Without question, this has been aided in part by the recent application of high-resolution tract tracing methodology in non-human primates (for review see Morecraft et al., 2009; Lanciego and Wouterlood, 2011). With razor sharp precision against very low levels of competing background artifact, we can now trace individual axons over long distances and localize eloquent immunoreactive terminal-like varicosities 0.5 $-3.5 \mu \mathrm{m}$ in diameter at the light microscopic level. In addition to the nearly exclusive contralateral projection that terminates primarily in laminae VII, IX and VI (Fig. 12A), our observations suggest that the M1 hand/arm projection to upper levels of the cervical enlargement heavily targets the dorsal and ventrolateral interneuron region of lamina VII, and preferentially the motoneuron pool forming the dorsal quadrants of lamina IX (Fig. 12B). In contrast, at inferior cervical levels, the projection to lamina VII preferentially innervates the same subsectors but diminishes in intensity whereas the projection to lamina IX is notably enhanced, targeting motoneuron regions located ventrally as well as dorsally (Fig. 12B). Throughout the cervical enlargement, a common pattern of labeling was also detected, reminiscent of the arrangement originally recognized by Kuypers (1982). At each cervical level a large projection was found in dorsomedial and dorsolateral regions of lamina VII, which extended ventrally through the ventrolateral subsector of lamina VII and into lamina IX of the ventral horn (Fig. 12B). Collectively, these unique arrangements of terminal labeling accentuate the complexity of the non-human primate M1 hand/arm corticospinal projection and are highly suggestive of divergent functional specialization at rostral and caudal regions of the cervical enlargement.

\section{Acknowledgments}

The first author would like to thank Dr. Gary Van Hoesen for the many thoughtful discussions of this topic and related issues, and for guidance, support and encouragement provided throughout his graduate studies and professional biomedical career.

GRANT SUPPORT: Supported by National Institutes of Health grant NS 046367. 


\section{REFERENCES}

Alstermark B, Isa T, Ohki Y, Saito Y. Disynaptic pyramidal excitation in forelimb motor neurons mediated via $\mathrm{C}_{3}-\mathrm{C}_{4}$ propriospinal neurons in the Macaca fuscata . J Neurophysiol. 1999; 82:35803585. [PubMed: 10601484]

Alstermark B, Pettersson LG, Nishimura Y, Yoshino-Saito K, Tsuboi, Takahashi M, Isa T. Motor command for precision grip in the macaque monkey can be mediated by spinal interneurons. $\mathrm{J}$ Neurophysiol. 2011; 106:122-126. [PubMed: 21511706]

Armand J. The origin, course and terminations of corticospinal fibers in various mammals. Prog Brain Res. 1982; 57:329-360. [PubMed: 7156398]

Armand J, Edgley SA, Lemon RN, Olivier E. Protracted postnatal development of corticospinal projections from the primary motor cortex to hand motoneurones in the macaque monkey. Exp Brain Res. 1994; 101:178-182. [PubMed: 7531155]

Armand J, Olivier E, Edgley SA, Lemon RN. Postnatal development of corticospinal projections from motor cortex to the cervical enlargement in the macaque monkey. J Neurosci. 1997; 17:251-266. [PubMed: 8987753]

Basbaum AI, Bautista DM, Scherrer G, Julius D. Cellular and molecular mechanisms of pain. Cell. 2009; 139:267-284. [PubMed: 19837031]

Biber MP, Kneisley LW, LaVail JH. Cortical neurons projecting to the cervical and lumbar enlargements of the spinal cord in young and adult rhesus monkeys. Exp Neurol. 1978; 59:492-508. [PubMed: 417943]

Bortoff GA, Strick PL. Corticospinal terminations in two new-world primates: further evidence that corticomotoneuronal connections provide part of the neural substrate for manual dexterity. $\mathrm{J}$ Neurosci. 1993; 13:5105-5118. [PubMed: 7504721]

Boudrias MH, Belhaj-Saiff A, Park MC, Cheney PD. Contrasting properties of motor output from the supplementary motor area and primary motor cortex in rhesus macaques. Cereb Cortex. 2006; 16:632-638. [PubMed: 16049188]

Boudrias MH, McPherson RL, Frost SB, Cheney PD. Output properties and organization of the forelimb representation of motor areas on the lateral aspect of the hemisphere in rhesus macaques. Cereb Cortex. 2010a; 20:169-186. [PubMed: 19561063]

Boudrias MH, Lee SP, Svojanovsky S, Cheney PD. Forelimb muscle representations and output properties of motor areas in the mesial wall of rhesus macaques. Cereb Cortex. 2010b; 20:704719. [PubMed: 19633176]

Brandt HM, Apkarian AV. Biotin-dextran: a sensitive anterograde tracer for neuroanatomic studies in rat and monkey. J Neurosci Meth. 1992; 45:35-40.

Brinkman J, Kuypers HG. Splitbrain monkeys: cerebral control of ipsilateral and contralateral arm, hand, and finger movements. Science. 1972; 176:536-539. [PubMed: 4624322]

Brinkman J, Kuypers HGJM. Cerebral control of contralateral and ipsilateral arm, hand and finger movements in the split-brain rhesus monkey. Brain. 1973; 96:653-674. [PubMed: 4204228]

Brochier T, Boudreau MJ, Paré M, Smith AM. The effects of muscimol inactivation of small regions of motor and somatosensory cortex on independent finger movements and force control in the precision grip. Exp Brain Res. 1999; 128:31-40. [PubMed: 10473737]

Bryan RN, Trevino DL, Willis WD. Evidence for a common location of alpha and gamma motoneurons. Brain Res. 1972; 38:193-196. [PubMed: 5015999]

Bucy PC. Representation of ipsilateral-extremities in the cerebral cortex. Science. 1933; 78:418. [PubMed: 17770479]

Bucy PC, Fulton JF. Ipsilateral representation in the motor and premotor cortex of monkeys. Brain. $1933 ; 56: 318-342$.

Catsman-Berrevoets CE, Kuypers HG. Cells of origin of corticospinal projections to dorsal column nuclei, spinal cord and bulbar medial reticular formation in the rhesus monkey. Neurosci Lett. 1976; 3:245-252. [PubMed: 19604894]

Cheney PD, Fetz EE, Mewes K. Neural mechanisms underlying corticospinal and rubrospinal control of limb movements. Prog Brain Res. 1991; 87:213-252. [PubMed: 1866448] 
Chiken S, Hatanaka N, Tokuno H. Distribution of median, ulnar and radial motoneurons in the monkey spinal cord: a retrograde triple-labeling study. Neurosci Lett. 2001; 307:143-146. [PubMed: 11438384]

Condé F. Further studies on the use of the fluorescent tracers fast blue and diamidino yellow: effective uptake area and cellular storage sites. J Neurosci Methods. 1987; 21:31-43. [PubMed: 3657270]

Coulter JD, Jones EG. Differential distribution of corticospinal projections from individual cytoarchitectonic fields in the monkey. Brain Res. 1977; 129:335-340. [PubMed: 69470]

Darling WG, Cole KJ. Muscle activation patterns and kinetics of human index finger movements. J Neurophysiol. 1990; 63:1098-1108. [PubMed: 2358864]

Darling WG, Pizzimenti MA, Morecraft RJ. Functional recovery following motor cortex lesions in non-human primates: experimental implications for human stroke patients. J Integr Neurosci. 2011; 10:353-384. [PubMed: 21960307]

Davidoff RA. The pyramidal tract. Neurology. 1990; 40:332-339. [PubMed: 2405296]

Dewald JP, Beer RF, Given JD, McGuire JR, Rymer WZ. Reorganization of flexion reflexes in the upper extremity of hemiparetic subjects. Muscle Nerve. 1999; 22:1209-1221. [PubMed: 10454716]

Dum RP, Strick PL. The origin of corticospinal projections from the premotor areas in the frontal lobe. J Neurosci. 1991; 11:667-689. [PubMed: 1705965]

Dum RP, Strick PL. Spinal cord terminations of the medial wall motor areas in macaque monkeys. J Neurosci. 1996; 16:6513-6525. [PubMed: 8815929]

Edwards, SB.; Hendrickson, A. The autoradiographic tracing of axonal connections in the central nervous system. In: Heimer, L.; RoBards, MJ., editors. Neuroanatomical tract-tracing methods. New York: Plenum Press; 1981. p. 171-205.

Evarts EV. Relation of pyramidal tract activity to force exerted during voluntary movement. $\mathbf{J}$ Neurophysiol. 1968; 31:14-27. [PubMed: 4966614]

Ferrier D. Experimental researches in cerebral physiology and pathology. West Riding Lunatic Asylum Med Report. 1873; 3:30-96.

Ferrier D, Yeo G. A record of experiments on the effects of lesions of different regions of the cerebral hemispheres. Philos Tran Royal Soc London. 1885; 175:479-564.

Fetz EE, Cheney PD, Mewes K, Palmer S. Control of forelimb muscle activity by populations of corticomotoneuronal and rubromotoneuronal cells. Prog Brain Res. 1989; 80:437-449. [PubMed: 2517459]

Fetz EE, Perlmutter SI, Prut Y, Seki K, Votaw S. Roles of primate spinal interneurons in preparation and execution of voluntary hand movement. Brain Res Rev. 2002; 40:53-65. [PubMed: 12589906]

Freese JL, Amaral DG. Synaptic organization of projections from the amygdala to visual cortical areas TE and V1 in the macaque monkey. J Comp Neurol. 2006; 496:655-667. 2006. [PubMed: 16615120]

Fritsch G, Hitzig E. Über die elektrische Erregbarkeit des Grosshirns. Arch Anat Physiol Wiss. 1870; 37:300-332.

Fulton JF. Spasticity and the frontal lobes. A review. New Eng J Med. 1937; 217:1017-1024.

Galea MP, Darian-Smith I. Multiple corticospinal neuron populations in the macaque monkey are specified by their unique cortical origins, spinal terminations, and connections. Cereb Cortex. 1994; 4:166-194. [PubMed: 8038567]

Galea MP, Darian-Smith I. Corticospinal projection patterns following unilateral section of the cervical spinal cord in the newborn and juvenile macaque monkey. J Comp Neurol. 1997; 381:282-306. [PubMed: 9133569]

Georgopoulos AP, Ashe J, Smyrnis N, Taira M. The motor cortex and the coding of force. Science. 1992; 256:1692-1695. [PubMed: 1609282]

Glaser, J.; Greene, G.; Hendricks, S. Stereology for biological research: with a focus on neuroscience. Williston VT: MBF Press; 2007.

Grandjean B, Hepp-Reymond MC, Maier MA. The functional role of different neural activation profiles during precision grip: an artificial neural network approach. J Physiol (Paris). 2007; 101:9-21. [PubMed: 18023563] 
Gundersen HJ, Bendtsen TF, Korbo L, Marcussen N, Moller A, Nielsen K, Nyengaard JR, Pakkenberg B, Sorensen FB, Vesterby A, West MJ. Some new, simple and efficient stereological methods and their use in pathological research and diagnosis. Acta Pathol Microbiol Immunol Scand. 1988; 96:379-394.

Haaxma R, Kuypers HG. Intrahemispheric cortical connexions and visual guidance of hand and finger movements in the rhesus monkey. Brain. 1975; 98:239-260. [PubMed: 1148818]

He SQ, Dum RP, Strick PL. Topographic organization of corticospinal projections from the frontal lobe: motor areas on the lateral surface of the hemisphere. J Neurosci. 1993; 13:952-980. [PubMed: 7680069]

He SQ, Dum RP, Strick PL. Topographic organization of corticospinal projections from the frontal lobe: motor areas on the medial surface of the hemisphere. J Neurosci. 1995; 15:3284-3306. [PubMed: 7538558]

Heffner R, Masterton R. Variation in form of the pyramidal tract and its relationship to digital dexterity. Brain Behav Evol. 1975; 12:161-200. [PubMed: 1212616]

Heffner RS, Masterton RB. The role of the corticospinal tract in the evolution of human dexterity. Brain Behav Evol. 1983; 23:165-183. [PubMed: 6667369]

Holstege, G. Brainstem-spinal cord projections in the cat, related to control of head and axial movements. In: Büttner-Ennever, JA., editor. Neuroanatomy of the occulomotor system. Amsterdam: Elsevier Science Publishers; 1988. p. 431-470.

Horsley V, Schäffer EA. A record of experiments upon the functions of the cerebral cortex. Philos Trans R Soc Lond, Ser B. 1888; 179:1-45.

Huntley GW, Jones EG. Relationship of intrinsic connections to forelimb movement representations in monkey motor cortex: a correlative anatomic and physiological study. J Neurophysiol. 1991; 66:390-413. [PubMed: 1723093]

Isa T, Ohki Y, Seki K, Alstermark B. Properties of propriospinal neurons in the $\mathrm{C}_{3}-\mathrm{C}_{4}$ segments mediating disynaptic pyramidal excitation to forelimb motoneurons in the macaque monkey. $\mathrm{J}$ Neurophysiol. 2006; 95:3674-3685. [PubMed: 16495365]

Isa T, Ohki Y, Alstermark B, Pettersson LG, Sasaki S. Direct and indirect cortico-motoneuronal pathways and control of hand/arm movements. Physiol. 2007; 22:145-153.

Jacobsen CF. Influence of motor and premotor area lesions upon the retention of acquired skilled movements in monkeys and chimpanzees. Res Pub Assoc Nerv Ment Dis. 1934; 13:225-247.

Jenny AB, Inukai J. Principles of motor organization of the monkey cervical spinal cord. J Neurosci. 1983; 3:567-575. [PubMed: 6827309]

Kennard MA, Fulton JF. The localizing significance of spasticity, reflex grasping, and the signs of Babinski and Rossolimo. Brain. 1933; 56:213-225.

Kinoshita M, Matsui R, Kato S, Hasegawa T, Kasahara H, Isa K, Watakabe A, Yamamori T, Nishimura Y, Alstermark B, Watanabe D, Kobayashi K, Isa T. Genetic dissection of the circuit for hand dexterity in primates. Nature. 2012; 487:235-238. [PubMed: 22722837]

Kucera $\mathrm{P}$, Wiesendanger M. Do ipsilateral corticospinal fibers participate in the functional recovery following unilateral pyramidal lesions in monkeys? Brain Res. 1985; 348:297-303. [PubMed: 4075087]

Kultas-Ilinsky K, Sivan-Loukianova E, Ilinsky IA. Reevaluation of the primary motor cortex connections with the thalamus in primates. J Comp Neurol. 2003; 457:133-158. [PubMed: 12541315]

Kuypers HGJM. Central cortical projections to motor and somato-sensory cell groups. An experimental study in the rhesus monkey. Brain. 1960; 83:161-184. [PubMed: 14413010]

Kuypers HGJM. The motor system and the capacity to execute highly fractionated distal extremity movements. Electroencephalogr Clin Neurophysiol Suppl. 1978; 34:429-431. [PubMed: 108079]

Kuypers, HGJM. Anatomy of the descending motor pathways. In: Brooks, VB., editor. Handbook of physiology, section I, The nervous system. Vol. Vol II. Bethesda: American Physiological Society; 1981. p. 567-666.Motor control

Kuypers HG. A new look at the organization of the motor system. Prog Brain Res. 1982; 57:381-403. [PubMed: 6818612] 
Kuypers HGJM, Brinknam J. Precentral projections to different parts of the spinal intermediate zone in the rhesus monkey. Brain Res. 1970; 24:29-48. [PubMed: 4099987]

Lanciego JL, Wouterlood FG. A half century of experimental neuroanatomical tracing. J Chem Neuroanat. 2011; 42:157-183. [PubMed: 21782932]

Lassek, AM. Its status in medicine. Springfield, Illinois: Charles C Thomas; 1954. The pyramidal tract.

Lawrence DG, Kuypers HGJM. The functional organization of the motor system in the monkey I. The effects of bilateral pyramidal lesions. Brain. 1968a; 91:1-14. [PubMed: 4966862]

Lawrence DG, Kuypers HGJM. The functional organization of the motor system in the monkey II. The effects of lesions of the descending brain-stem pathways. Brain. 1968b; 91:15-36. [PubMed: 4966860]

Lawrence DG, Porter R, Redman SJ. Corticomotoneuronal synapses in the monkey: light microscopic localization upon motoneurons of intrinsic muscles of the hand. J Comp Neurol. 1985; 232:499 510. [PubMed: 3980765]

Leichnetz GR. Afferent and efferent connections of the dorsolateral precentral gyrus (area 4, hand/arm region) in the macaque monkey, with comparisons to area 8. J Comp Neurol. 1986; 254:460-492. [PubMed: 3805358]

Lemon RN. Cortical control of the primate hand. Exp Physiol. 1993; 78:263-301. [PubMed: 8329205]

Lemon RN. Descending pathways in motor control. Annu Rev Neurosci. 2008; 31:195-218. [PubMed: 18558853]

Lemon RN, Griffiths J. Comparing the function of the corticospinal system in different species: organizational differences for motor specialization? Muscle Nerve. 2005; 32:261-279. [PubMed: 15806550]

Lemon RN, Kirkwood PA, Maier MA, Nakajima K, Nathan P. Direct and indirect pathways for corticospinal control of upper limb motoneurons in the primate. Prog Brain Res. 2004; 143:263279. [PubMed: 14653171]

Lemon RN, Landau W, Tutssel D, Lawrence DG. Lawrence and Kuypers (1968a, b) revisited: copies of the original filmed material from their classic papers in Brain. Brain. 2012; 135:2290-2295. [PubMed: 22374938]

Lemon RN, Maier MA, Armand J, Kirkwood PA, Yang HW. Functional differences in corticospinal projections from macaque primary motor cortex and supplementary motor area. Adv Exp Med Biol. 2002; 508:425-434. [PubMed: 12171139]

Leyton ASF, Sherrington CS. Observations on the excitable cortex of the chimpanzee, orang-utan and gorilla. Q J Exp Physiol. 1917; 11:135-222.

Liu CN, Chambers WW. An experimental study of the cortico-spinal system in monkey (Macaca mulatta). The spinal pathways and preterminal distribution of degenerating fibers following discrete lesions of the pre- and postcentral gyri and bulbar pyramid. J Comp Neurol. 1964; 123:257-283. [PubMed: 14219668]

Luppino G, Matelli M, Camarda R, Rizzolatti G. Corticospinal projections from mesial frontal and cingulate areas in the monkey. Neuroreport. 1994; 5:2545-2548. [PubMed: 7696600]

Maier MA, Armand J, Kirkwood PA, Yang HW, Davis JN, Lemon RN. Differences in the corticospinal projection from primary motor cortex and supplementary motor area to macaque upper limb motoneurons: an anatomical and electrophysiological study. Cereb Cortex. 2002; 12:281-296. [PubMed: 11839602]

Maier MA, Bennett KM, Hepp-Reymond MC, Lemon RN. Contribution of the monkey corticomotoneuronal system to the control of force in precision grip. J Neurophysiol. 1993; 69:772-785. [PubMed: 8463818]

Martin JH. The corticospinal system: from development to motor control. The Neuroscientist. 2005; 11:161-173. [PubMed: 15746384]

McNeal DW, Darling WG, Ge J, Stilwell-Morecraft KS, Solon KM, Hynes SM, Pizzimenti MA, Rotella DL, Vanadurongvan T, Morecraft RJ. Selective long-term reorganization of the corticospinal projection from the supplementary motor cortex following recovery from lateral motor cortex injury. J Comp Neurol. 2010; 518:586-621. [PubMed: 20034062] 
Mesulam MM. Tetramethyl benzidine for horseradish peroxidase neurohistochemistry: a noncarcinogenic blue reaction-product with superior sensitivity for visualizing neural afferents and efferents. J Histochem Cytochem. 1978; 26:106-117. [PubMed: 24068]

Mesulam, MM. Tracing neural connections with horseradish peroxidase. New York: John Wiley and Sons; 1982.

Mollazadeh M, Aggarwal V, Davidson AG, Law AJ, Thakor NV, Schieber MH. Spatiotemporal variation of multiple neurophysiological signals in the primary motor cortex during dexterous reach-to-grasp movements. J Neurosci. 2011; 31:15531-15543. [PubMed: 22031899]

Morecraft RJ, Cipolloni PB, Stilwell-Morecraft KS, Gedney MT, Pandya DN. Cytoarchitecture and cortical connections of the posterior cingulate and adjacent somatosensory fields in the rhesus monkey. J Comp Neurol. 2004; 469:37-69. [PubMed: 14689472]

Morecraft RJ, Herrick JL, Stilwell-Morecraft KS, Louie JL, Schroeder CM, Ottenbacher JG, Schoolfield MW. Localization of arm representation in the corona radiata and internal capsule in the non-human primate. Brain. 2002; 125:176-198. [PubMed: 11834603]

Morecraft RJ, Geula C, Mesulam MM. Cytoarchitecture and neural afferents of orbitofrontal cortex in the brain of the monkey. J Comp Neurol. 1992; 323:341-358. [PubMed: 1460107]

Morecraft RJ, McNeal DW, Stilwell-Morecraft KS, Dvanajscak Z, Ge J, Schneider P. Localization of arm representation in the cerebral peduncle of the non-human primate. J Comp Neurol. 2007a; 504:149-167. [PubMed: 17626268]

Morecraft RJ, McNeal DW, Stilwell-Morecraft KS, Gedney M, Ge J, Schroeder CM, Van Hoesen GW. Amygdala interconnections with the cingulate motor cortex in the rhesus monkey. J Comp Neurol. 2007b; 500:134-165. [PubMed: 17099887]

Morecraft RJ, Stilwell-Morecraft KS, Cipolloni PB, Ge J, McNeal DW, Pandya DN. Cytoarchitecture and cortical connections of the anterior cingulate and adjacent somatomotor fields in the rhesus monkey. Brain Res Bull. 2012; 87:457-497. [PubMed: 22240273]

Morecraft, RJ.; Ugollini, G.; Lanciego, JL.; Wouterlood, FG.; Pandya, DN. Classic and contemporary neural tract tracing techniques. In: Johansen-Berg, H.; Behrens, T., editors. Diffusion MRI: from quantitative measurement to in-vivo neuroanatomy. Oxford: Elsevier; 2009. p. 273-308.

Morecraft RJ, Van Hoesen GW. Cingulate input to the primary and supplementary motor cortices in the rhesus monkey: evidence for somatotopy in areas 24c and 23c. J Comp Neurol. 1992; 322:471-489. [PubMed: 1383283]

Morecraft RJ, Van Hoesen GW. Frontal granular cortex input to the cingulate (M3), supplementary (M2) and primary (M1) motor cortices in the rhesus monkey. J Comp Neurol. 1993; 337:669-689. [PubMed: 8288777]

Morris R, Cheunsuang O, Stewart A, Maxwell D. Spinal dorsal horn neurone targets for nociceptive primary afferents: do single neurone morphological characteristics suggest how nociceptive information is processed at the spinal level. Brain Res Rev. 2004; 46:173-190. [PubMed: 15464206]

Mouton LJ, Holstege G. The periaqueductal gray in the cat projects to lamina VIII and the medial part of lamina VII throughout the length of the spinal cord. Exp Brain Res. 1994; 101:253-264. [PubMed: 7531157]

Mullen RJ, Buck CR, Smith AM. NeuN, a neuronal specific nuclear protein in vertebrates. Develoment. 1992; 116:201-211.

Murray EA, Coulter JD. Organization of corticospinal neurons in the monkey. J Comp Neurol. 1981; 195:339-365. [PubMed: 7251930]

Nudo RJ, Masterton RB. Descending pathways to the spinal cord, III: sites of origin of the corticospinal tract. J Comp Neurol. 1990; 296:559-583. [PubMed: 2113540]

Park MC, Belhaj-Saïf A, Gordon M, Cheney PD. Consistent features in the forelimb representation of primary motor cortex in rhesus monkeys. J Neurosci. 2001; 21:2784-2792. [PubMed: 11306630]

Park MC, Belhaj-Saif A, Cheney PD. Properties of primary motor cortex output to forelimb muscles in rhesus macaques. J Neurophysiol. 2004; 92:2968-2984. [PubMed: 15163675]

Phillips CG, Porter R. The pyramidal projection to motoneurones of some muscle groups of the baboon's forelimb. Prog Brain Res. 1964; 12:222-245. [PubMed: 14202441] 
Pizzimenti MA, Darling WG, Rotella DL, McNeal DW, Herrick JL, Ge J, Stilwell-Morecraft KS, Morecraft RJ. Measurement of reaching kinematics and prehensile dexterity in nonhuman primates. J Neurophysiol. 2007; 98:1015-1029. [PubMed: 17553948]

Porter, R.; Lemon, R. Corticospinal functions and voluntary movement. Oxford: Clarendon Press; 1993.

Ralston DD, Ralston HJ. The terminations of corticospinal tract axons in the macaque monkey. J Comp Neurol. 1985; 242:325-337. [PubMed: 2418074]

Rathelot J-A, Strick PL. Muscle representation in the macaque motor cortex: an anatomical perspective. Proc Natl Acad Sci. 2006; 103:8257-8262. [PubMed: 16702556]

Rathelot J-A, Strick PL. Subdivisions of primary motor cortex based on cortico-motoneuronal cells. Proc Natl Acad Sci. 2009; 106:918-923. [PubMed: 19139417]

Reiner, A.; Honig, MG. Dextran amines: versatile tools for anterograde and retrograde studies of nervous system connectivity. In: Zaborszky, L.; Wouterlood, FG.; Lanciego, JL., editors. Neuroanatomical tract-tracing: molecules, neurons and systems. Vol. 3. New York: Springer; 2006. p. 304-335.

Reiner A, Veenman CL, Honig MG. Anterograde tracing using biotinylated dextran amine. Neurosci Protocols. 1993; 93 section 050-14.

Rexed B. A cytoarchitectonic atlas of the spinal cord in the cat. J Comp Neurol. 1954; 100:297-379. [PubMed: 13163236]

Richter CP, Hines M. Experimental production of the grasp reflex in adult monkeys by lesions of the frontal lobes. Amer J Physiol. 1932; 101:87-88.

Richter CP, Hines M. The production of the 'grasp reflex' in adult macaques by experimental frontal lobe lesions. Res Publ Assoc Nerv Ment Dis. 1934; 12:211.

Rosenzweig ES, Brock JH, Culbertson MD, Lu P, Moseanko R, Edgerton VR, Havton LA, Tuszynski MH. Extensive spinal decussation and bilateral termination of cervical corticospinal projections in rhesus monkeys. J Comp Neurol. 2009; 513:151-163. [PubMed: 19125408]

Rouiller EM, Moret V, Tanne J, Boussaoud D. Evidence for direct connections between the hand region of the supplementary motor area and cervical motoneurons in the macaque monkey. Eur $\mathbf{J}$ Neurosci. 1996; 8:1055-1059. [PubMed: 8743753]

Sasaki S, Isa T, Pettersson LG, Alstermark B, Naito K, Yoshimura K, Seki K, Ohki Y. Dexterous finger movements in primate without monosynaptic corticomotoneuronal excitation. $\mathrm{J}$ Neurophysiol. 2004; 92:3142-3147. [PubMed: 15175371]

Schieber MH. Comparative anatomy and physiology of the corticospinal system. Handb Clin Neurol. 2007; 82:15-37. [PubMed: 18808887]

Schieber MH. Dissociating motor cortex from the motor. J Physiol. 2011; 589:5613-5624. [PubMed: 22005673]

Schieber MH, Poliakov AV. Partial inactivation of the primary motor cortex hand area: effects on individuated finger movements. J Neurosci. 1998; 18:9038-9054. [PubMed: 9787008]

Schieber MH, Santello M. Hand function: peripheral and central constraints on performance. J Appl Physiol. 2004; 96:2293-2300. [PubMed: 15133016]

Schmahmann, JD.; Pandya, DN. Fiber pathways of the brain. Oxford: Oxford University Press; 2006.

Schmahmann JD, Rosene DL, Pandya DN. Motor projections to the basis pontis in the rhesus monkey. J Comp Neurol. 2004; 478:248-268. [PubMed: 15368534]

Schofield BR. Uptake of Phaseolus vulgaris leucoagglutinin (PHA-L) by axons of passage. J Neurosci Methods. 1990; 35:47-56. [PubMed: 1703615]

Shalit U, Zinger N, Joshua M, Prut Y. Descending systems translate transient cortical commands into a sustained muscle activation signal. Cereb Cortex. 2012; 22:1904-1914. [PubMed: 21965441]

Sherrington CS. On nerve-tracts degenerating secondarily to lesions of the cortex cerebri. J Physiol. $1889 ; 10: 429-432$.

Soteropoulos DS, Edgley SA, Baker SN. Lack of evidence for direct corticospinal contributions to control of the ipsilateral forelimb in monkey. J Neurosci. 2011; 31:11208-11219. [PubMed: 21813682] 
Strick PL, Burke RE, Kanda K, Kim CC, Walmsley B. Differences between alpha and gamma motoneurons labeled with horseradish peroxidase by retrograde transport. Brain Res. 1976; 113:582-588. [PubMed: 60166]

Takei T, Seki K. Spinal interneurons facilitate coactivation of hand muscles during a precision grip task in monkeys. J Neurosci. 2010; 30:17041-17050. [PubMed: 21159974]

Todd AJ. Neuronal circuitry for pain processing in the dorsal horn. Nat Rev Neurosci. 2010; 11:823836. [PubMed: 21068766]

Travis, AM. University of Wisconsin; 1952. Neurological deficits produced by precentral and supplementary motor area lesions. Thesis

Travis AM. Neurological deficiencies following supplementary motor area lesions in macaca mulatta. Brain. 1955; 78:174-198. [PubMed: 13239907]

Warr, WB.; de Olmos, JS.; Heimer, L. Horseradish peroxidase: the basic procedure. In: Heimer, L.; RoBards, MJ., editors. Neuroanatomical tract-tracing methods. New York: Plenum Press; 1981. p. 207-262.

West, MJ. Basic stereology for biologists and neuroscientists. New York: Cold Spring Harbor Laboratory Press; 2012.

Wiesendanger M. Postlesion recovery of motor and sensory cortex in the early twentieth century. $\mathrm{J}$ Hist Neurosci. 2011; 20:42-57. [PubMed: 21253939]

Wise, SP. Corticospinal efferents of the supplementary sensorimotor area in relation to the primary motor area. In: Lüders, HO., editor. Advances in neurology. Vol. Vol. 70. supplementary sensorimotor area. Philadelphia: Lippincott-Raven Publishers; 1996. p. 57-69.

Wolf HK, Buslei R, Schmidt-Kastner R, Schmidt-Kastner PK, Pietsch T, Wiestler OD, Blümcke I. NeuN: a useful neuronal marker for diagnostic histopathology. J Histochem Cytochem. 1996; 44:1167-1171. [PubMed: 8813082]

Wouterlood FG, Groenewegen HJ. Neuroanatomical tracing by use of Phaseolus vulgarisleucoagglutinin (PHA-L): electron microscopy of PHA-L-filled neuronal somata, dendrites, axons and axon terminals. Brain Res. 1985; 326:188-191. [PubMed: 3882193]

Wu G, Perlmutter SI. Sensitivity of spinal neurons to GABA and glycine during voluntary movement in behaving monkeys. J Neurophysiol. 2013; 109:193-201. [PubMed: 23076104]

$\mathrm{Xu} \mathrm{Q}$, Grant G. Do certain spinocerebellar neurons in lamina IX at lumbosacral levels send collaterals to peripheral nerves? A retrograde fluorescent double labeling study in the cat. Arch Ital Biol. 1988; 126:179-192. [PubMed: 3178393]

Yanai Y, Adamit N, Harel R, Israel Z, Prut Y. Connected corticospinal sites show enhanced tuning similarity at the onset of voluntary action. J Neurosci. 2007; 27:12349-12357. [PubMed: 17989299]

Yeterian EH, Van Hoesen GW. Cortico-striate projections in the rhesus monkey: the organization of certain cortico-caudate connections. Brain Res. 1978; 139:43-63. [PubMed: 413609]

Yoshino-Saito K, Nishimura Y, Oishi T, Isa T. Quantitative inter-segmental and inter-laminar comparison of corticospinal projections from the forelimb area of the primary motor cortex of macaque monkeys. Neurosci. 2010; 171:1164-1179.

Zaaimi B, Edgley SA, Soteropoulos DS, SN Baker. Changes in descending motor pathway connectivity after corticospinal tract lesion in macaque monkey. Brain. 2012; 135:2277-2289. [PubMed: 22581799]

Zinger N, Harel R, Gabler S, Israel Z, Prut Y. Functional organization of information flow in the corticospinal pathway. J Neurosci. 2013; 33:1190-1197. [PubMed: 23325255]

\section{LIST OF ABBREVIATIONS}

$\begin{array}{ll}\text { BDA } & \text { Biotinylated Dextran Amine } \\ \text { CSP } & \text { Corticospinal Projection } \\ \text { D } & \text { Dorsal }\end{array}$




$\begin{array}{ll}\text { DL } & \text { Dorsolateral subdivision } \\ \text { DM } & \text { Dorsomedial } \\ \text { L } & \text { Lateral } \\ \text { LCST } & \text { Lateral corticospinal tract } \\ \text { LYD } & \text { Lucifer yellow dextran } \\ \text { M } & \text { Medial } \\ \text { M1 } & \text { Primary Motor Cortex } \\ \text { M2 } & \text { Supplementary Motor Cortex } \\ \text { RMB } & \text { Reticulated Marginal Border } \\ \text { ROI } & \text { Region of Interest } \\ \text { V } & \text { Ventral, or ventral subdivision of lamina VII } \\ \text { VL } & \text { Ventrolateral subdivision } \\ \text { VM } & \text { Ventromedial subdivision } \\ \text { Xc } & \text { Contralateral subdivision of lamina X } \\ \text { Xi } & \text { Ipsilateral subdivision of lamina X } \\ \text { I-X } & \text { Rexed's laminae }\end{array}$



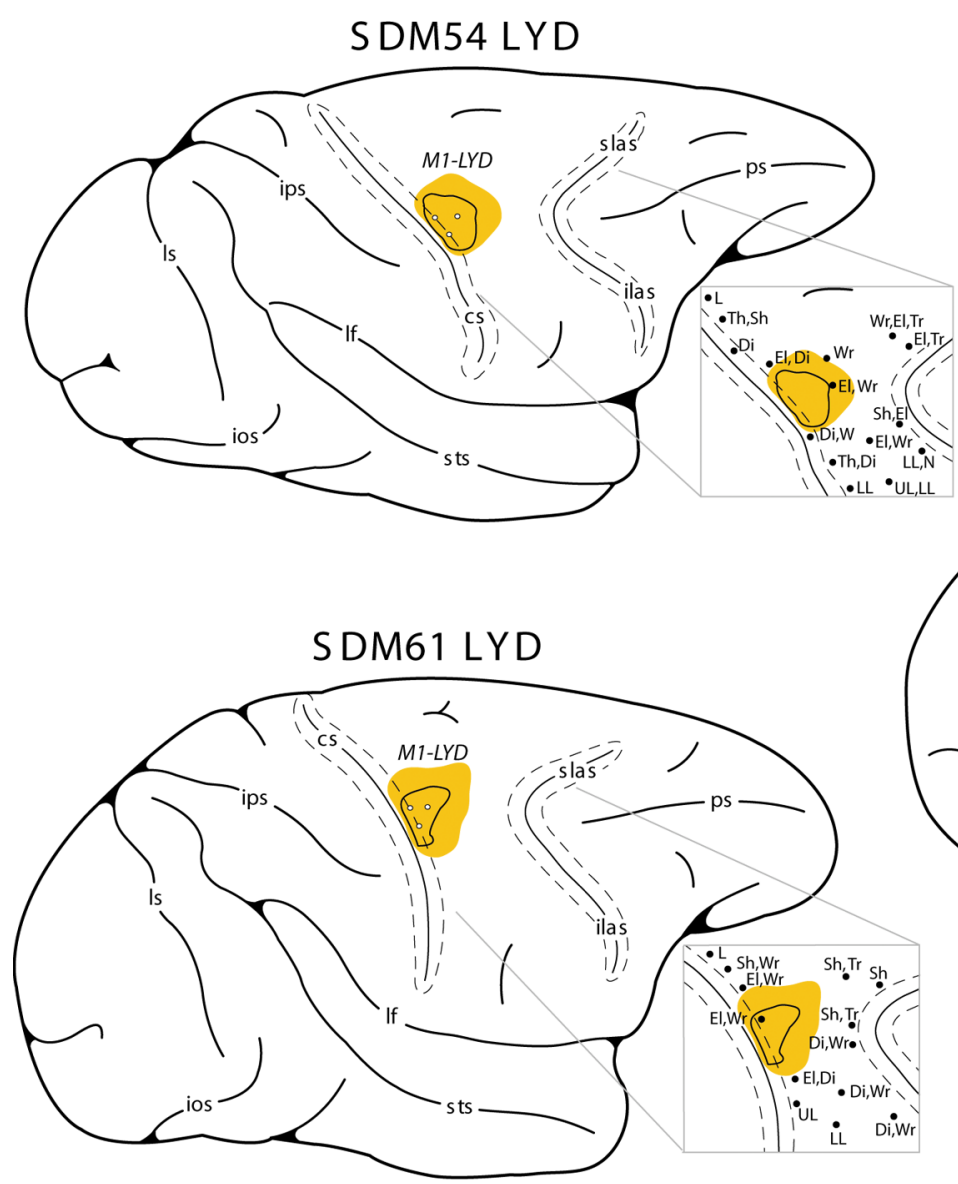

SDM57 LYD

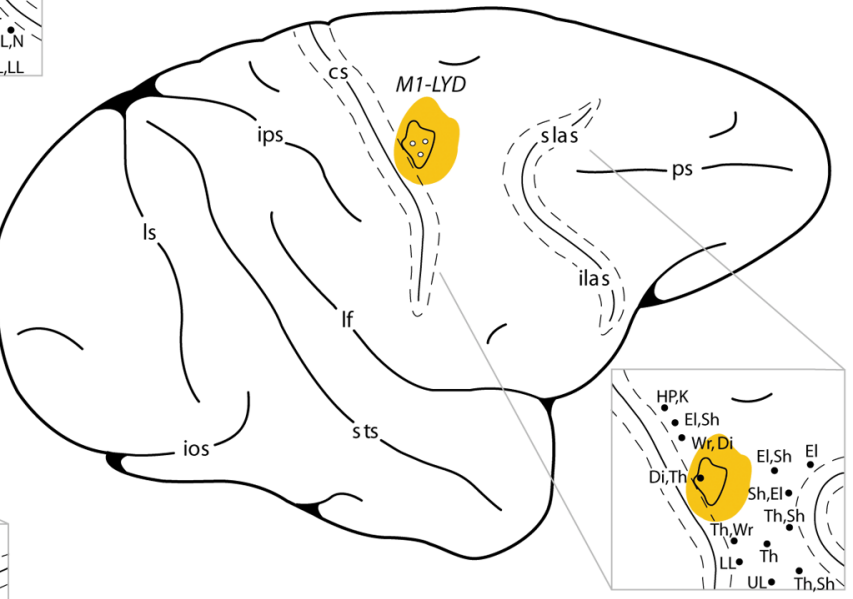

SDM62 BDA

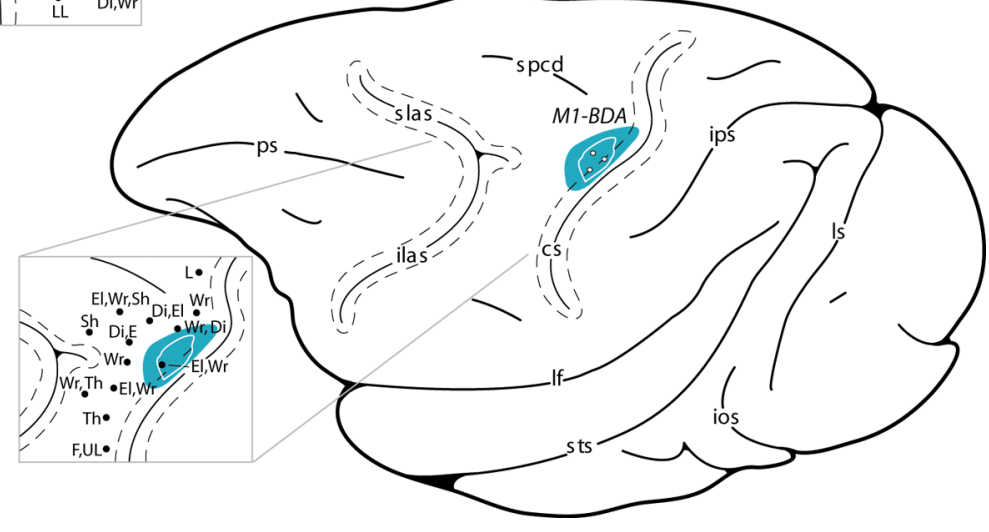

Figure 1.

Line drawings of the lateral surface of the cerebral cortex in cases SDM54, SDM57, SDM61 and SDM62 depicting the respective lucifer yellow dextran (LYD) and biotinylated dextran amine (BDA) injection sites into the cortical hand/arm region of M1. On the full lateral views, the irregular shaped black line within the yellow colored LYD injection sites and irregular white shaped line within the blue colored BDA injection site represents the boundary between the centrally located injection site core, and peripherally located injection site halo. The three small white dots located within the core region represent the Hamilton needle penetration sites through which each respective tract tracer was injected. The pullout depicts the physiological map of movement representation obtained with intracortical microstimulation used to localize the hand/arm representation of M1 prior to injection of each respective tract tracer. Abbreviations: cs, central sulcus; Di, digit; EL, elbow; F, face; HP, hip; ilas, inferior limb of arcuate sulcus; ios, inferior occipital sulcus; ips, intraparietal 
sulcus; K, knee; L, leg; ls, lunate sulcus; lf, lateral fissure; LL, lower lip; M1, primary motor cortex; ps, principle sulcus; SH, shoulder; slas, superior limb of the arcuate sulcus; spcd, superior pre-central dimple; sts, superior temporal sulcus; Th, thumb; UL, upper lip; W, wrist. 

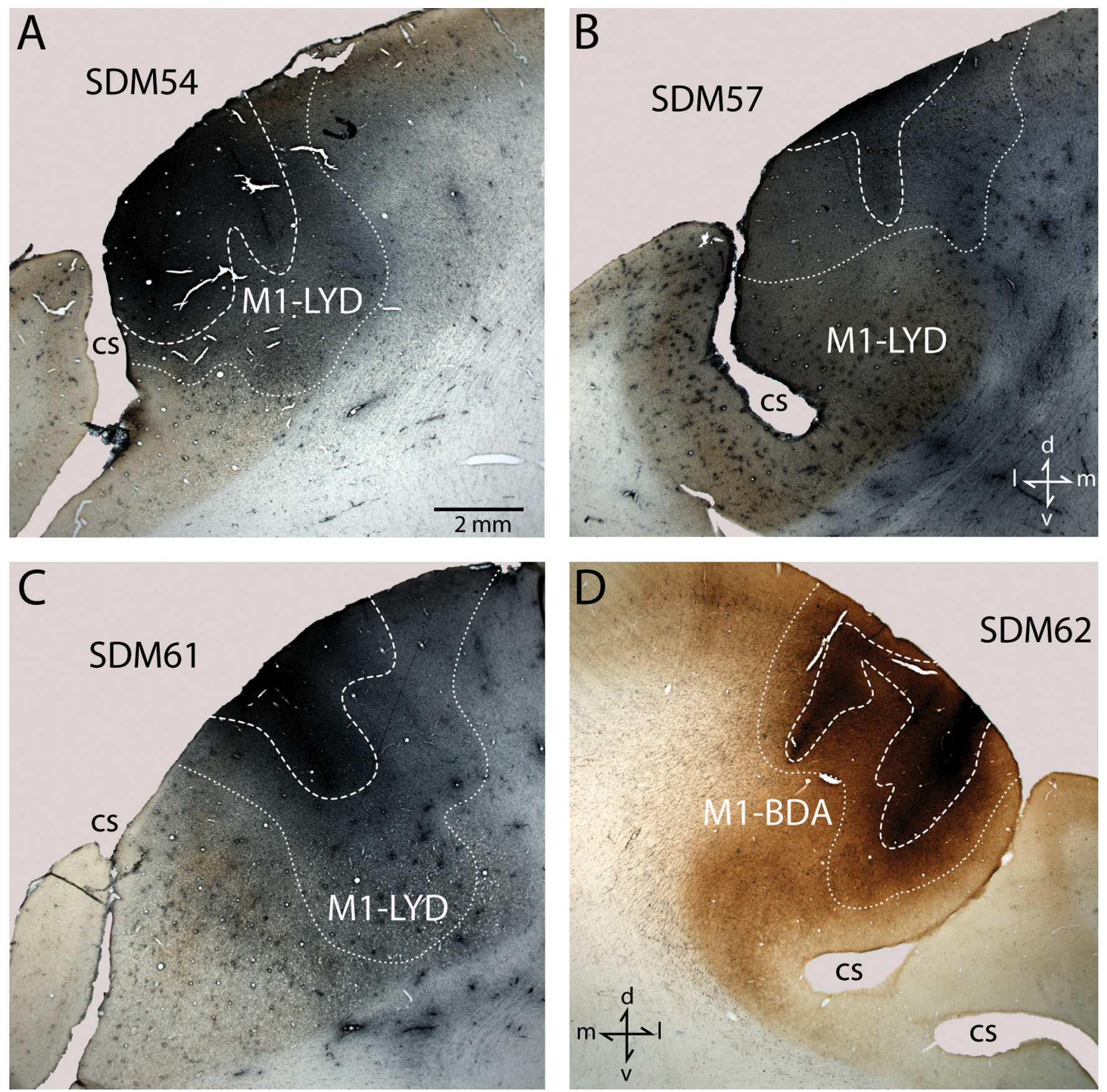

Figure 2.

Plate of low-power digital photomicrographs of immunohistochemically processed coronal tissue sections through the cerebral cortex illustrating the respective lucifer yellow dextran (LYD) and biotinylated dextran amine (BDA) injection sites in cases SDM54-LYD (A), SDM57-LYD (B), SDM61-BDA (C) and SDM62-LYD (D). On the injection site, the dashed line represents the external boundary of the injection site core and the dotted line the external boundary of the injection site halo which was determined using microscopic analysis. The scale bar in A applies to all panels. The anatomical orientation compass in panel B also applies to panels A and C. Abbreviations: cs, central sulcus; d, dorsal; 1, lateral; $\mathrm{m}$, medial; v, ventral. 

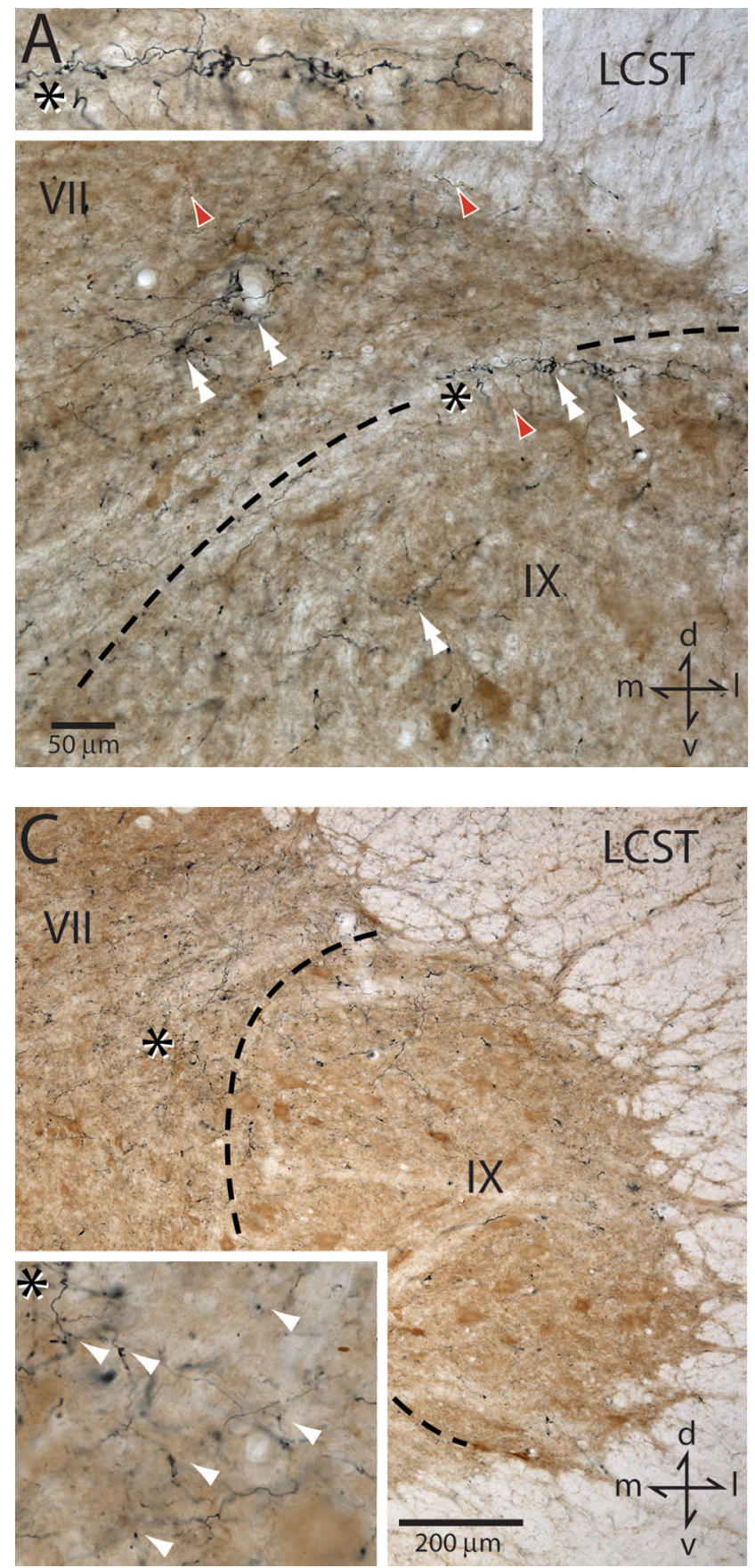

Figure 3.
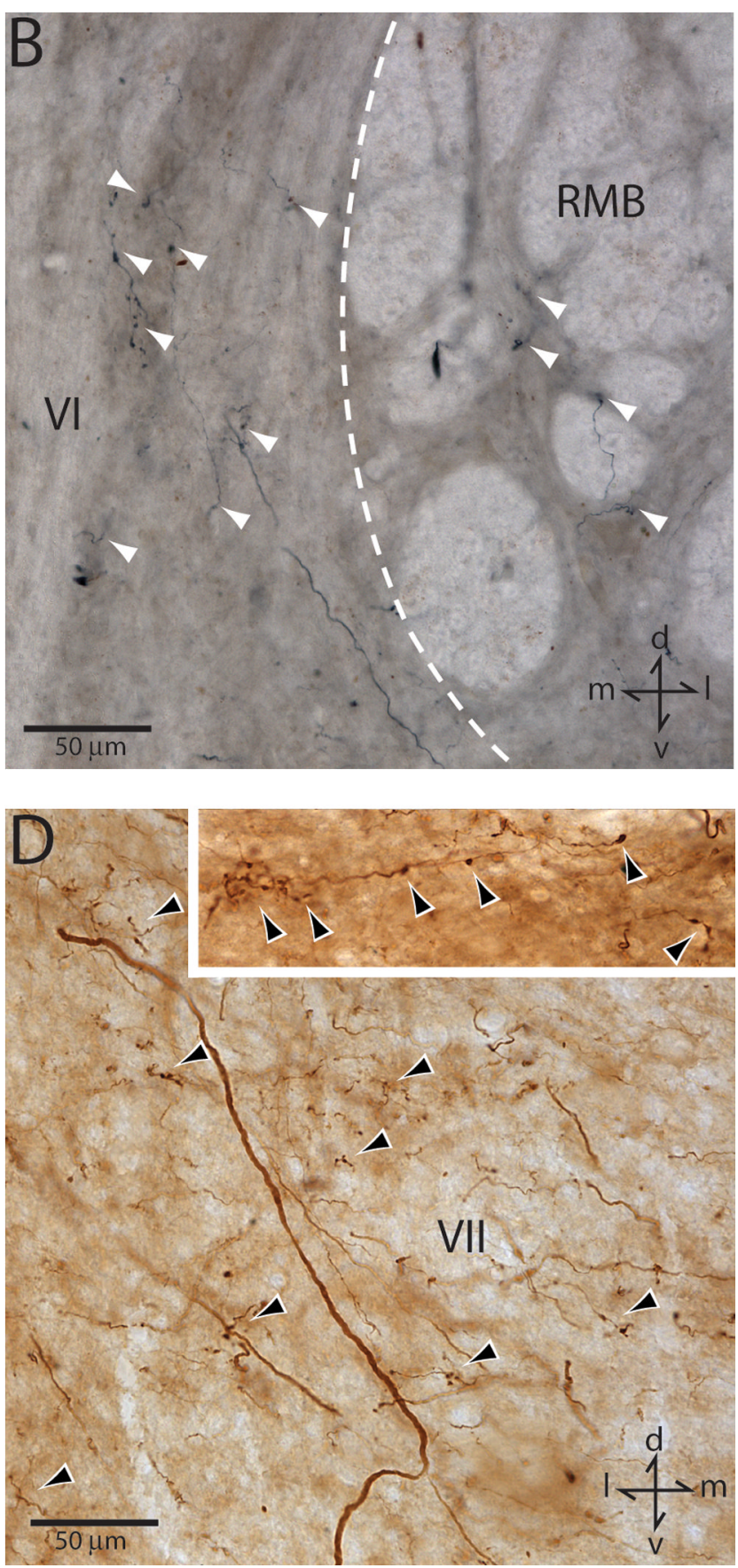

Plate of high-power digital photomicrographs of immunohistochemically processed tissue sections through the spinal cord illustrating dextran labeled terminal axon fibers and boutons (arrowheads) in the gray matter of cases SDM54-LYD (A, spinal level C8), SDM57-LYD (B, spinal level C6), SDM61-LYD (C, spinal level T1) and SDM62-BDA (D, spinal level C5). LYD terminal fibers and boutons were visualized using the blue reaction product procedure and BDA fibers and terminals the brown reaction product procedure (see Materials and Methods). In panel A the white double arrowheads indicate sites of intense LYD-labeled bouton clusters (blue reation product) and the red arrowheads identify some 
brown colored BDA labeled fibers and boutons resulting from a BDA injection site placed into another cortical region of interest. In $\mathrm{A}$ and $\mathrm{C}$ the inset is a high power view of the field marked by the asterisk. In panel D, the inset shows a high power view of labeled terminals (arrowheads) in the dorsolateral region of lamina IX at C8. Roman numerals represent Rexed's laminae. Abbreviations: d, dorsal; 1, lateral; LCST, lateral corticospinal tract; m, medial; RMB, reticulated marginal border; v, ventral. 


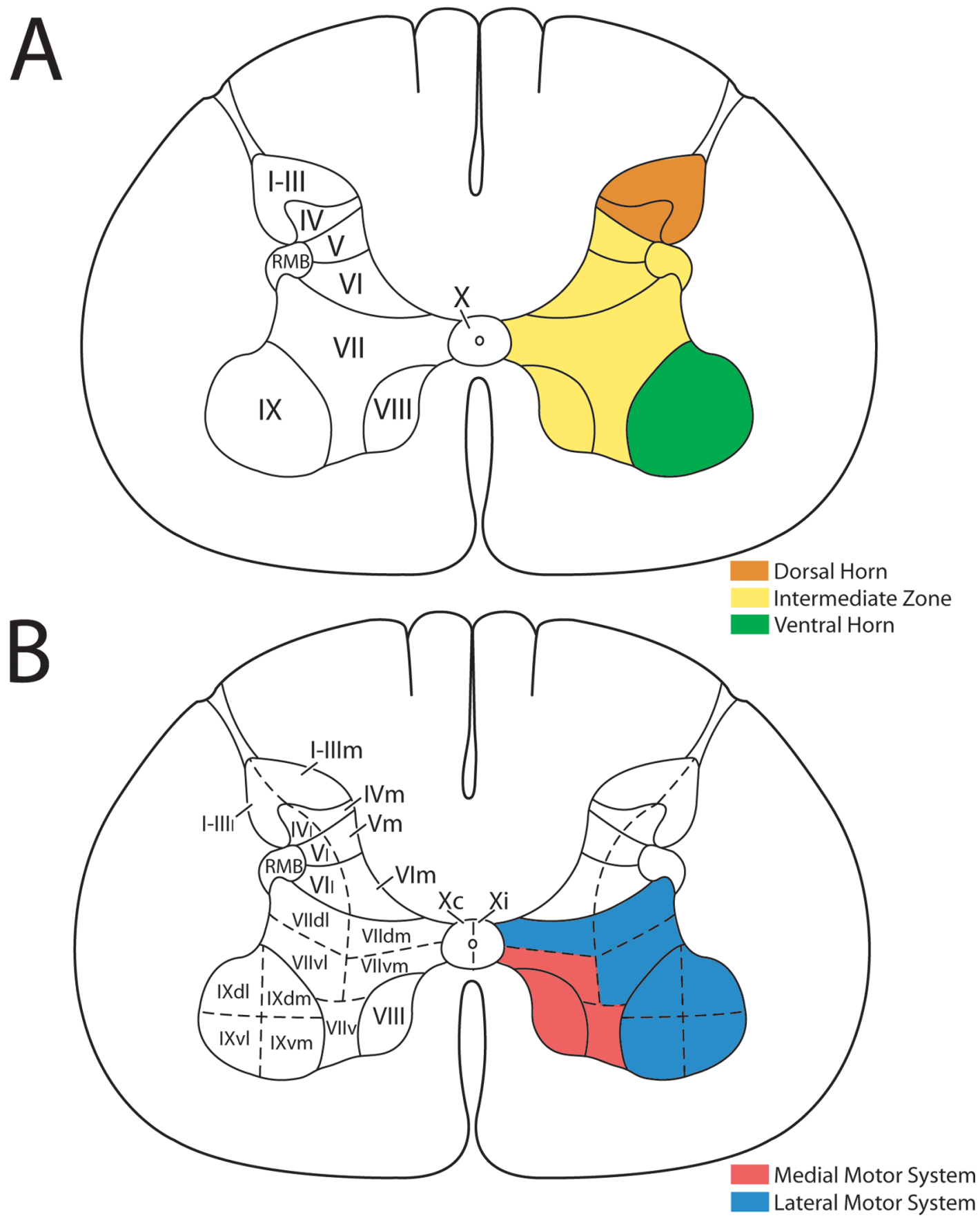

Figure 4.

For this study, the general anatomical organization of the spinal cord was adopted from Kuypers (1981). Thus, in panel A, the ventral horn consisted of lamina IX (green shading) the intermediate zone laminae V, VI, VII, VIII and the RMB (yellow shading), and the dorsal horn laminae I-III and VI (orange shading). In panel B, left, we further subdivided Rexed's laminae into 20 subdivisions (regions of interest) in each half of the spinal cord. In panel B, right, the blue shaded regions of lamina VII and IX represent spinal components of Kuypers (1982) "lateral motor system" (see diagonal hatching and circles on the horizontal spinal cord section in Fig. 9 of Kuypers, 1982). The red shaded region of lamina VII and lamina VIII constitute parts of his "medial motor system" " (see light stippling on the 
horizontal spinal cord section in Fig. 9 of Kuypers, 1982). For abbreviations see text under "Anatomical Nomenclature" and "List of Abbreviations". 

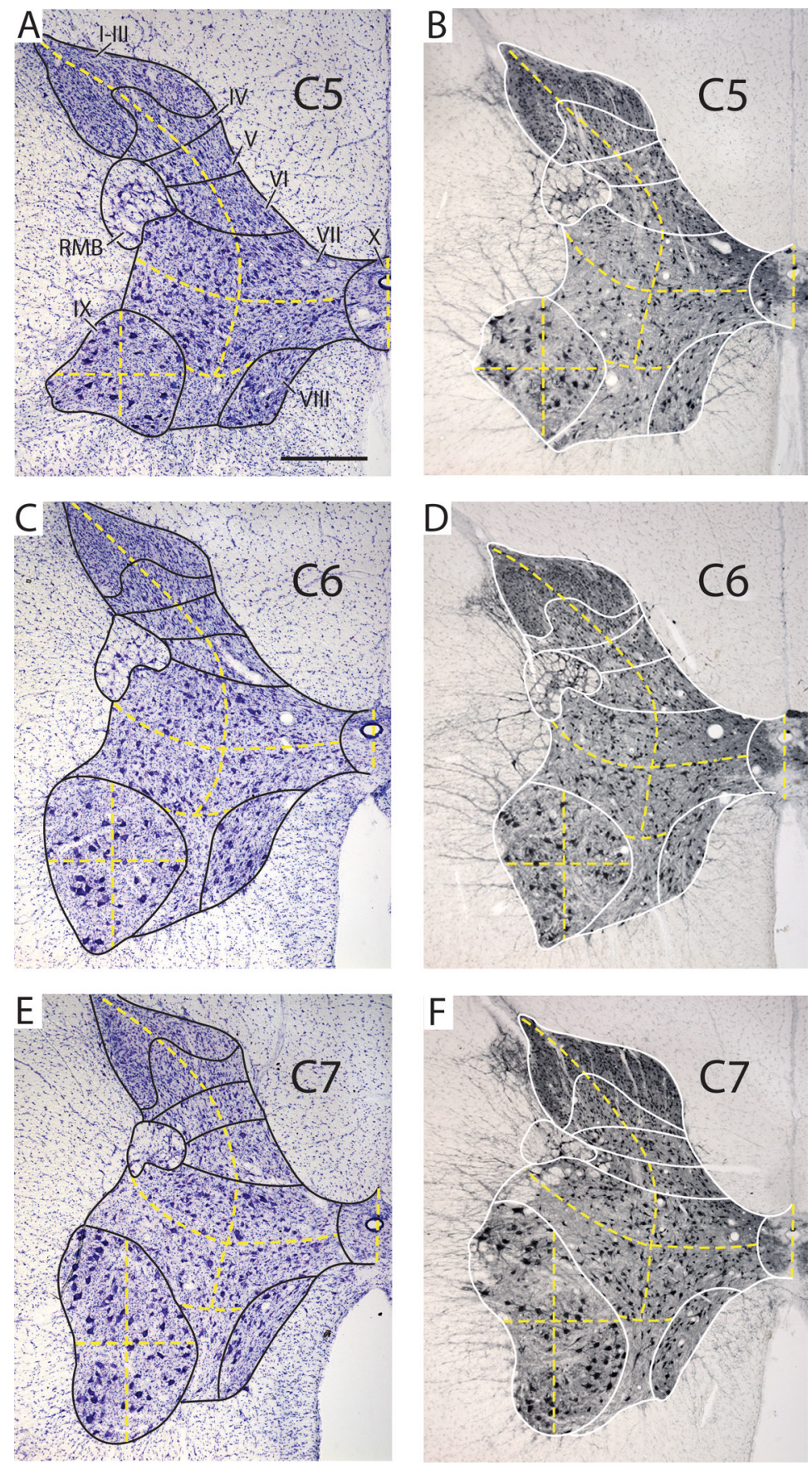

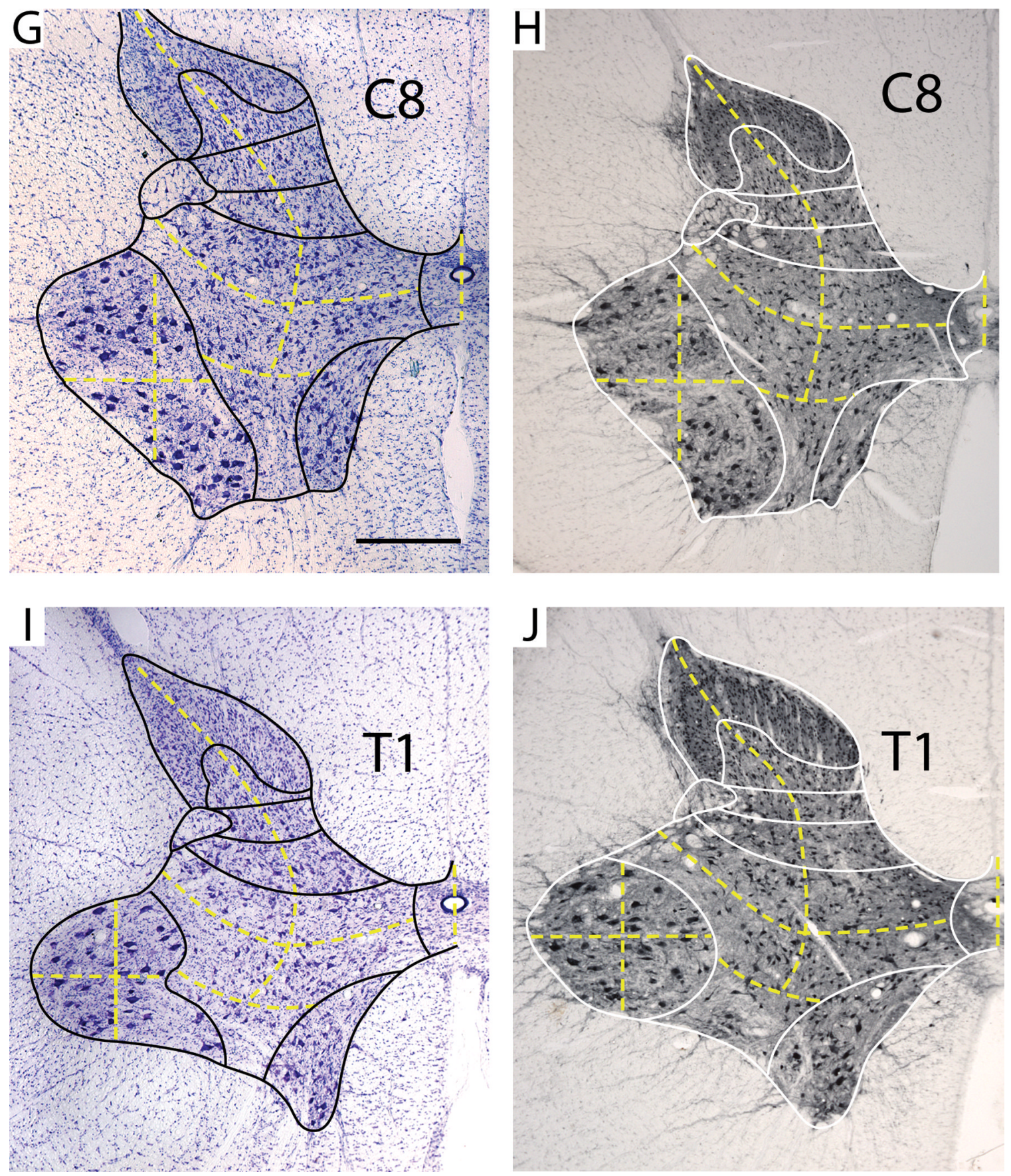

Figure 5.

Representative horizontal Nissl stained tissue sections (panels A, C, E, G, I) for each spinal level (C5, C6, C7, C8, T1) and corresponding NeuN stained tissue sections (panels B, D, F, $\mathrm{H}, \mathrm{J}$ ) for each level. On both sets of tissue sections the general boundaries of Rexed's laminae are indicated by the solid lines and the various subsectors within each lamina are indicated by the dashed lines (see Fig. 4B, for subdivision identification). Scale bar in panels $\mathrm{A}$ and $\mathrm{J}$ is $500 \mu \mathrm{m}$ and applies to all other panels. 

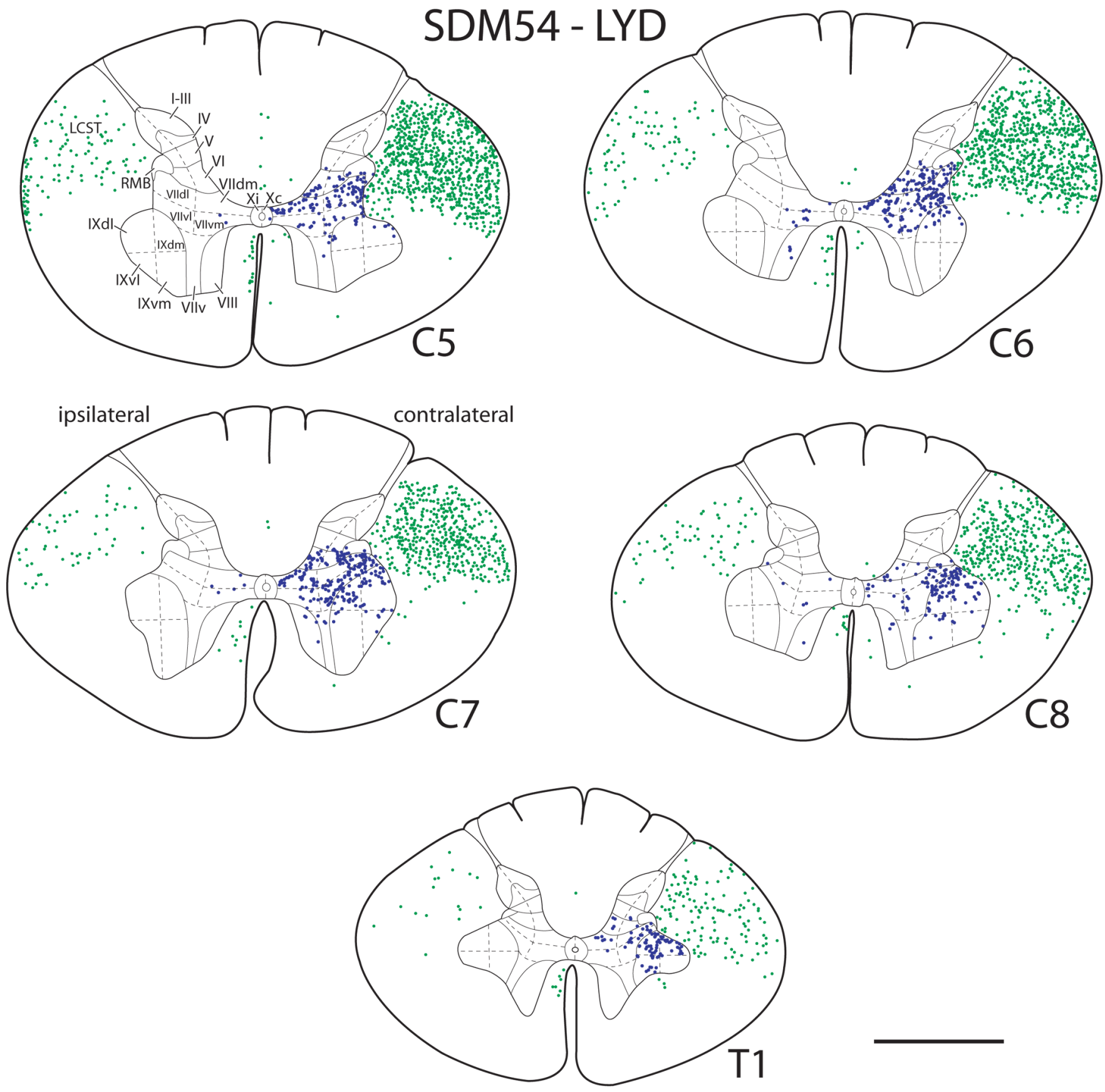

Figure 6.

Line drawings depicting selected horizontal sections with dense labeling through spinal levels C5 to T1 in case SDM54-LYD. Labeled axons in the lateral corticospinal tract and other white matter regions are identified by the green dots and terminal boutons and bouton clusters within Rexed's laminae and reticulated marginal border (RMB) are identified by the blue dots. Roman numerals in section C5 designate Rexed's laminae and their respective subdivisions and apply to all spinal sections. Micron bar $=2 \mathrm{~mm}$. For abbreviations see text under "Anatomical Nomenclature" or "List of Abbreviations". 

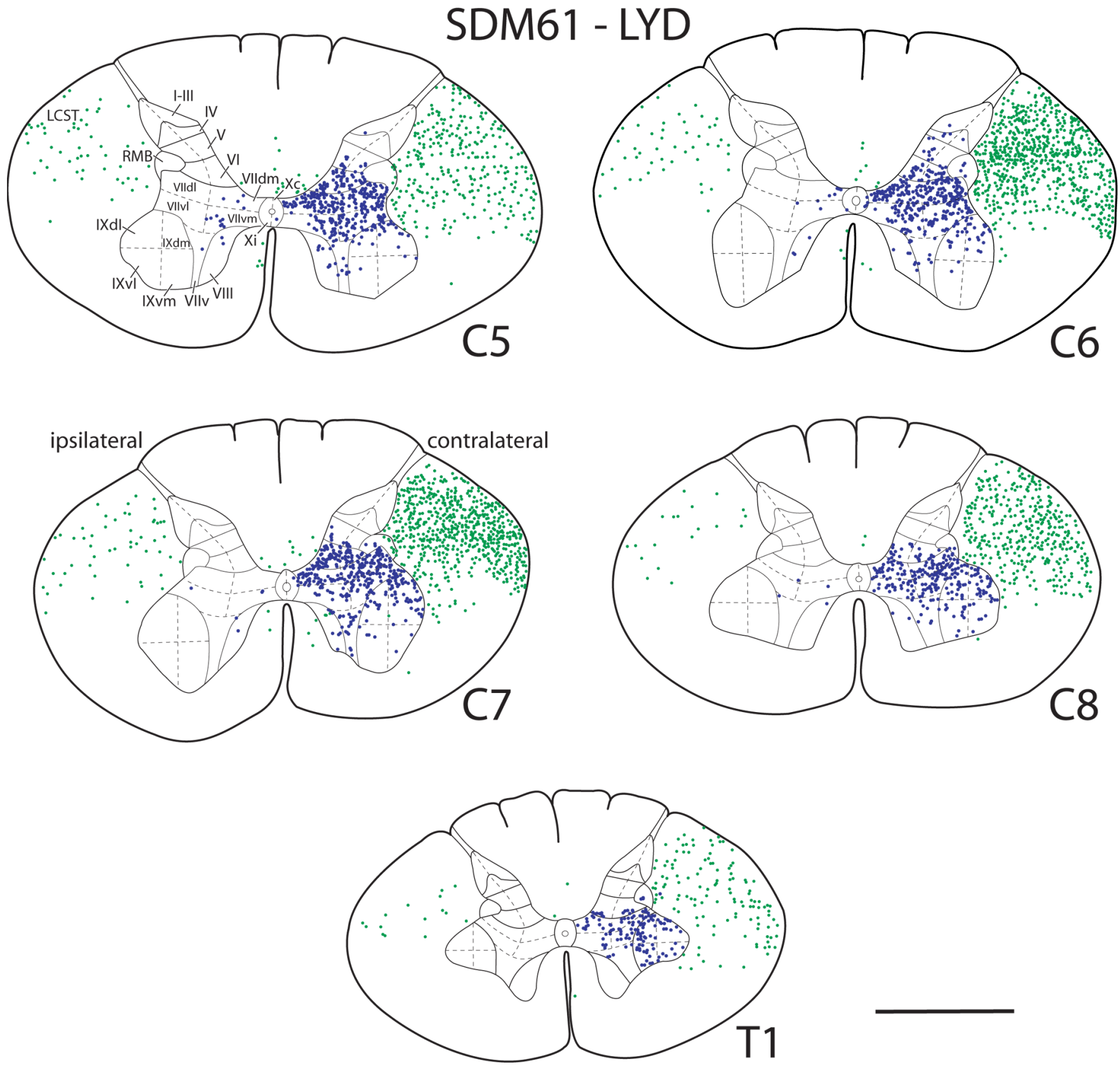

Figure 7.

Line drawings depicting selected horizontal sections with dense labeling through spinal levels C5 to T1 in case SDM61-LYD. Labeled axons in the lateral corticospinal tract and other white matter regions are identified by the green dots and terminal boutons and bouton clusters within Rexed's laminae are identified by the blue dots. Roman numerals in section C5 designate Rexed's laminae and their respective subdivisions and apply to all spinal sections. Micron bar $=2 \mathrm{~mm}$. For abbreviations see text under "Anatomical Nomenclature" or "List of Abbreviations". 

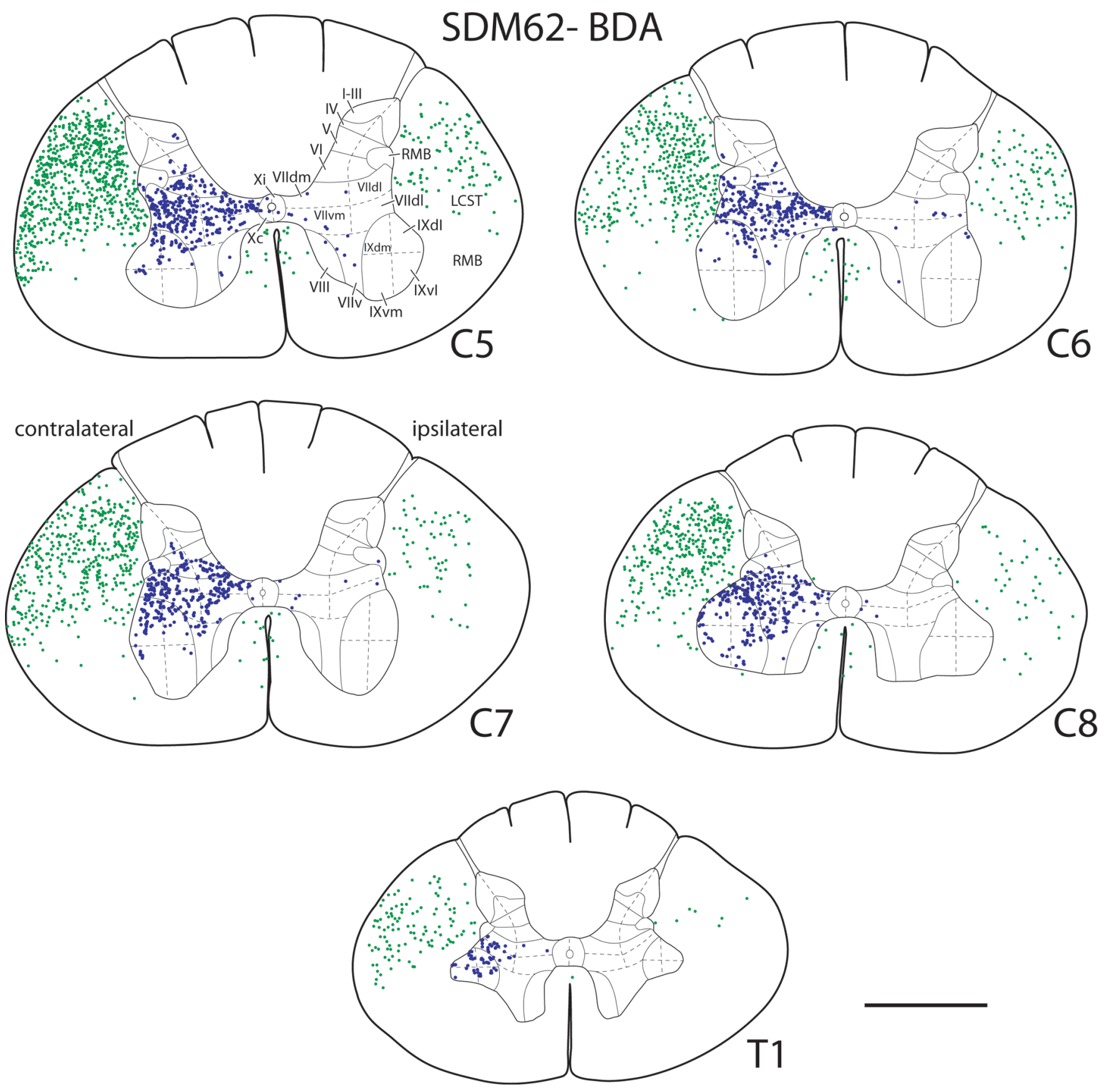

Figure 8.

Line drawings depicting selected horizontal sections with dense labeling through spinal levels C5 to T1 in case SDM62-BDA. Labeled axons in the lateral corticospinal tract and other white matter regions are identified by the green dots and terminal boutons and bouton clusters within Rexed's laminae are identified by the blue dots. Roman numerals in section C5 designate Rexed's laminae and their respective subdivisions and apply to all spinal sections. Micron bar $=2 \mathrm{~mm}$. For abbreviations see text under "Anatomical Nomenclature" or "List of Abbreviations". 

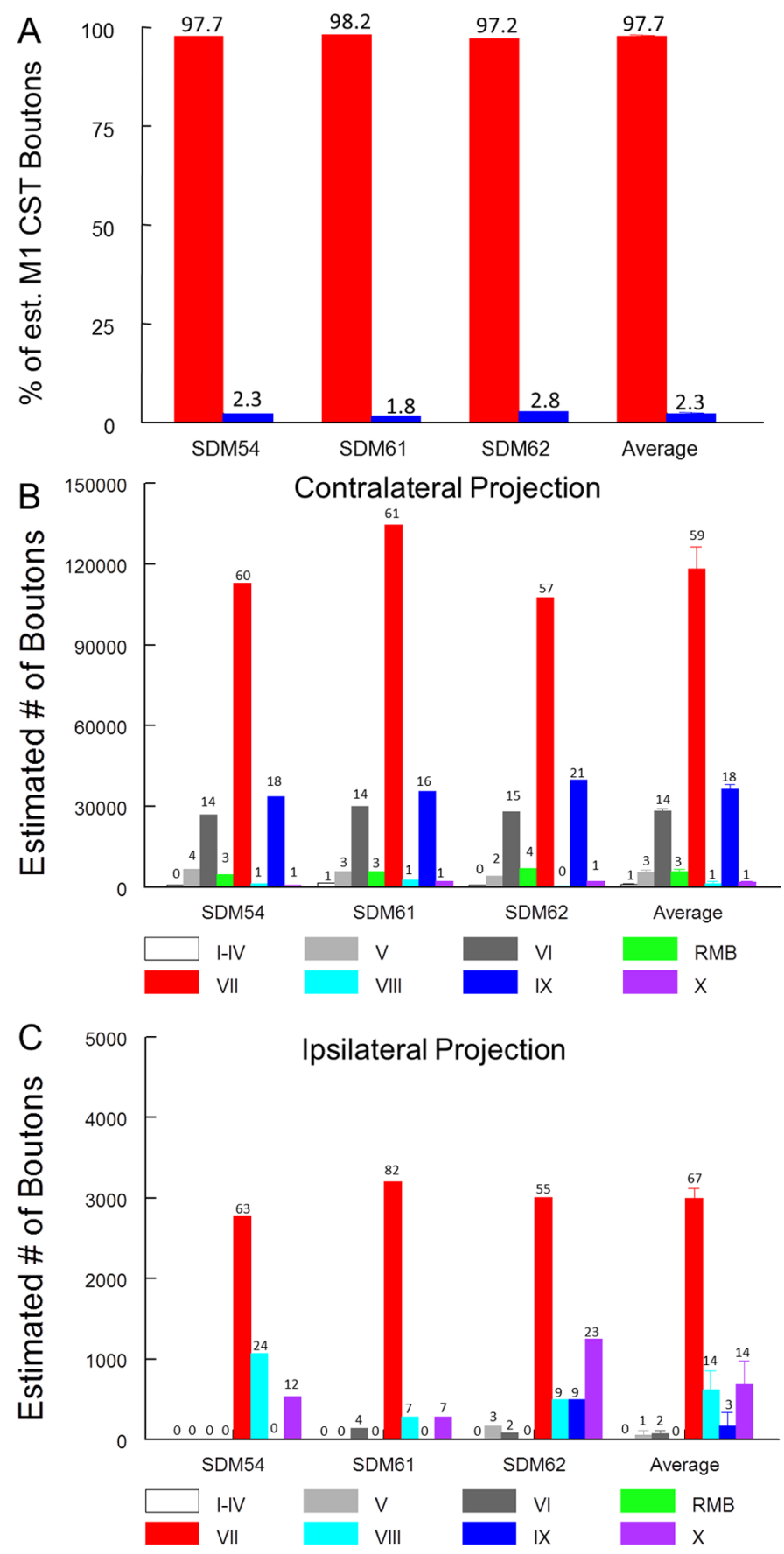

Figure 9.

A) Percentage of total estimated boutons distributed in the contralateral (red bars) and ipsilateral (blue bars) spinal laminae in segments C5 - T1. Each bar is the percentage of boutons from each individual monkey (SDM54, SDM61, SDM62) and the average of all three monkeys (far right) as indicated on the abscissa. Almost $98 \%$ of all labeled boutons were located in the contralateral spinal cord. Error bars on the average data set (far right) are 1 SEM. Estimated number of contralateral (B) and ipsilateral (C) boutons within Rexed's lamina of C5 - T1 in each individual monkey case and the average of three monkeys as indicated on the abscissa. Each bar represents the stereological estimate of the total number 
of boutons in the dorsal horn (lamina I-IV), intermediate zone (laminae V, VI, VII and VIII and RMB), ventral horn (lamina IX) and centrally located lamina X in C5 - T1. The number above each bar represents the percentage of all contralateral (B) or ipsilateral (C) boutons located in each lamina (I-X) or region (RMB) for each monkey case and for the average obtained from all three monkeys. Note the different ordinate scales in B and C. Error bars on the average data are 1 SEM. 

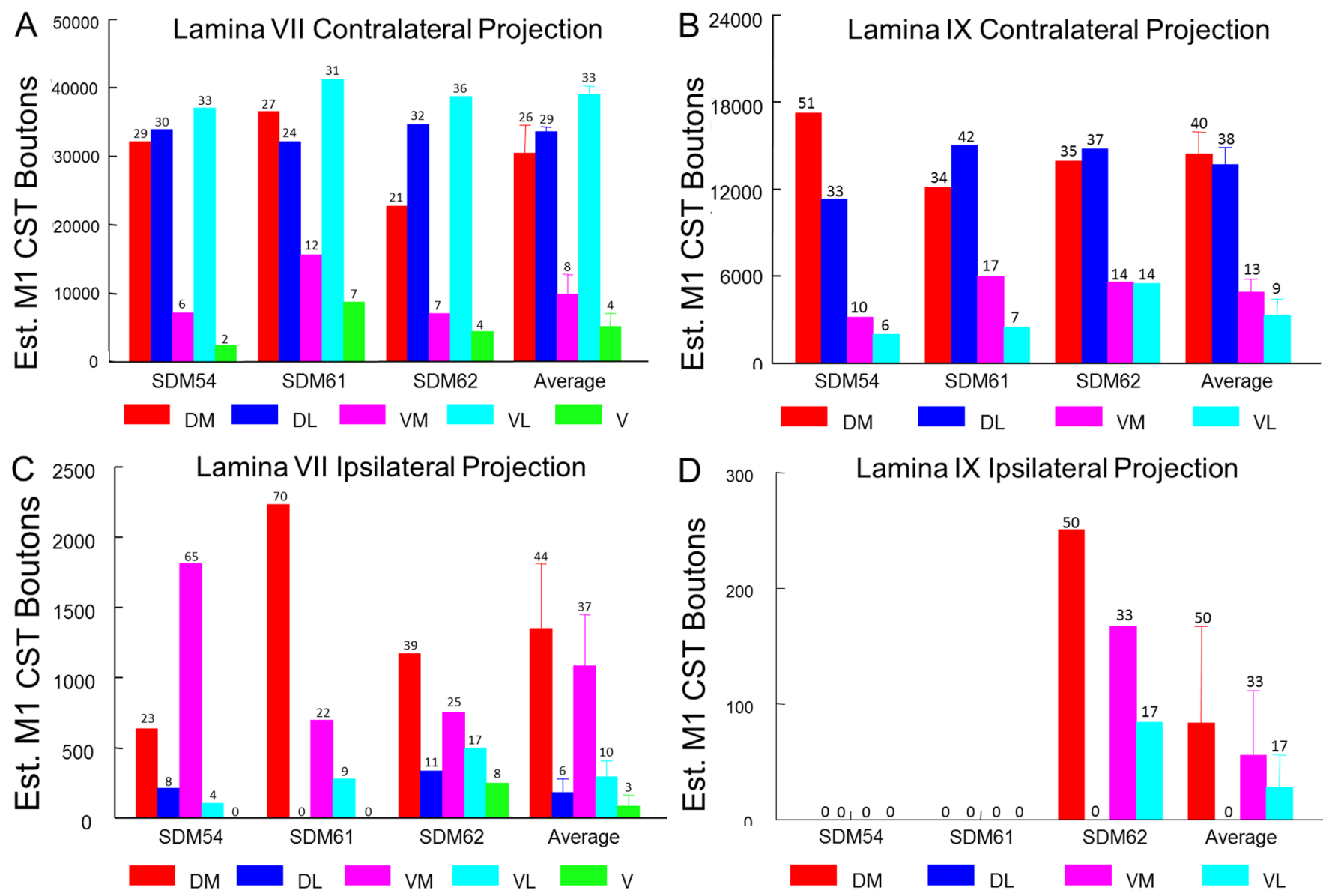

Figure 10.

A) Estimated number of contralateral boutons within each subsector of lamina VII in C5 T1 for each individual monkey (SDM54, SDM61, SDM62) and the average of all three (far right) as indicated on the abscissa. B) Estimated number of contralateral boutons within each subsector of lamina IX in C5 - T1 in each individual monkey case and the average of all three monkeys as indicated on the abscissa. C) Estimated number of ipsilateral boutons within each subsector of lamina VII in C5 - T1 for each individual monkey and the average of all three as indicated on the abscissa. D) Estimated number of ipsilateral boutons within each subsector of lamina IX in C5 - T1 in each individual monkey and the average of all three monkeys as indicated on the abscissa. Note the different ordinate scales in A and B (contralateral projection) versus $\mathrm{C}$ and $\mathrm{D}$ (ipsilateral projection). In each panel, the number above each bar is the percentage of either all contralateral or ipsilateral boutons located lamina VII and IX for that individual case or for the computed average obtained from all three monkeys. Error bars on the average data sets in all panels are 1 SEM. Abbreviations: DM, dorsomedial; DL, dorsolateral; VM, ventromedial; VL, ventrolateral; V, ventral. 

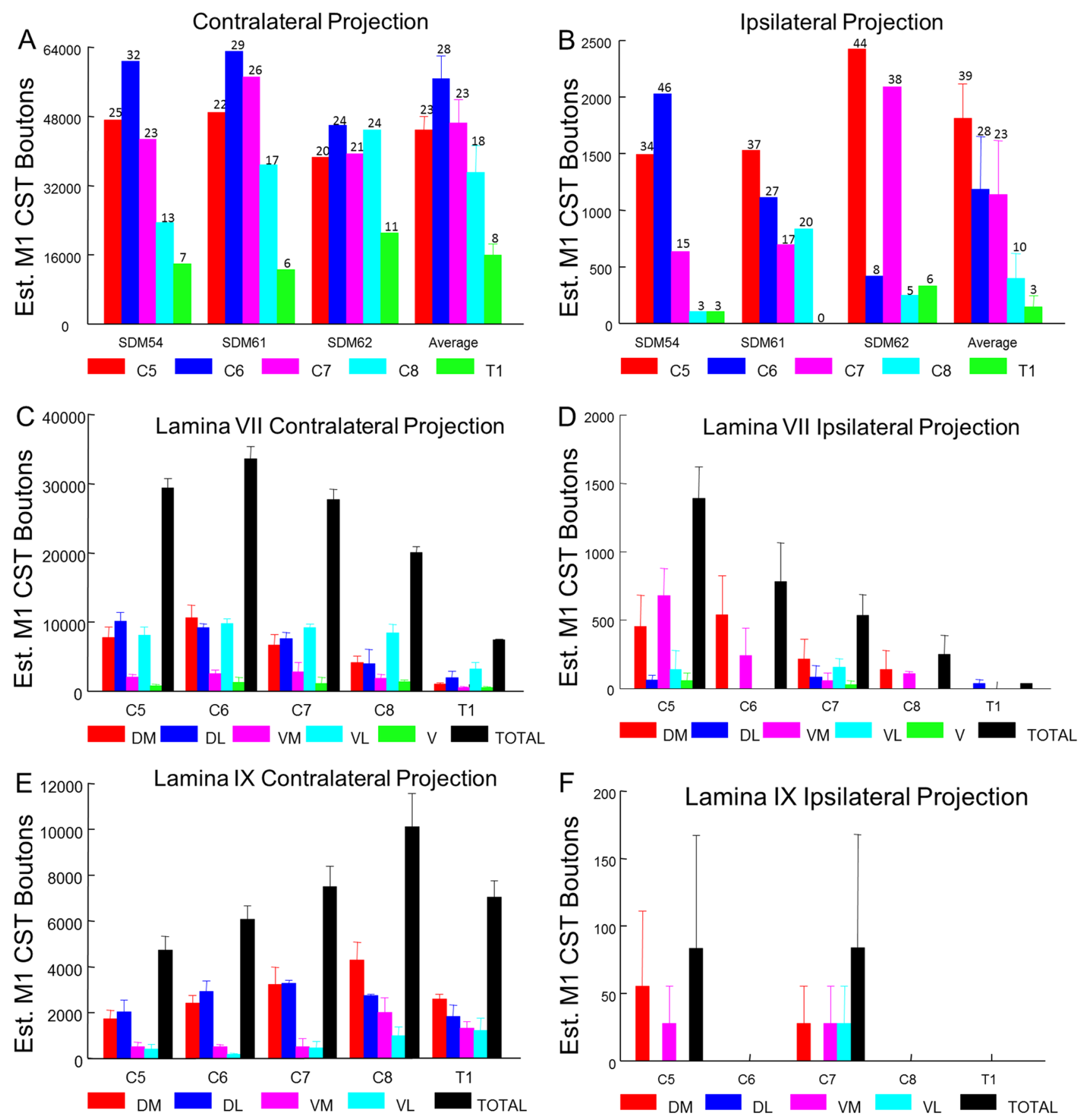

Figure 11.

Graphs showing the data obtained from our segmental analysis. Estimated number of contralateral (A) and ipsilateral (B) boutons in all laminae of each spinal segment (C5 - T1). Each bar is the stereological estimate of the number of boutons in all lamina of each spinal segment (C5 - T1) from each individual monkey (SDM54, SDM61, SDM62) and the average of all three monkeys (far right) as indicated on the abscissae. The number above each bar represents the percentage of boutons for that monkey or average percentage from all three monkeys. Estimated number of contralateral (C) and ipsilateral (D) boutons in the various subsectors of lamina VII of each spinal segment (C5 - T1). The black bars represent 
the average total number of boutons for each segmental level. Note the decline in total number of boutons progressing caudally. Estimated number of contralateral (E) and ipsilateral (F) boutons in the various subsectors of lamina IX of each spinal segment (C5 T1). The black bars represent the average total number of boutons for each segmental level. Note the gradual increase in total number of boutons from $\mathrm{C} 5$ to $\mathrm{C} 8$. Note the different ordinate scales in contralateral (A, C and D) and ipsilateral (B, D and F) projections. Error bars on the average data sets are 1 SEM. Abbreviations: DM, dorsomedial; DL, dorsolateral; VM, ventromedial; VL, ventrolateral; V, ventral. 

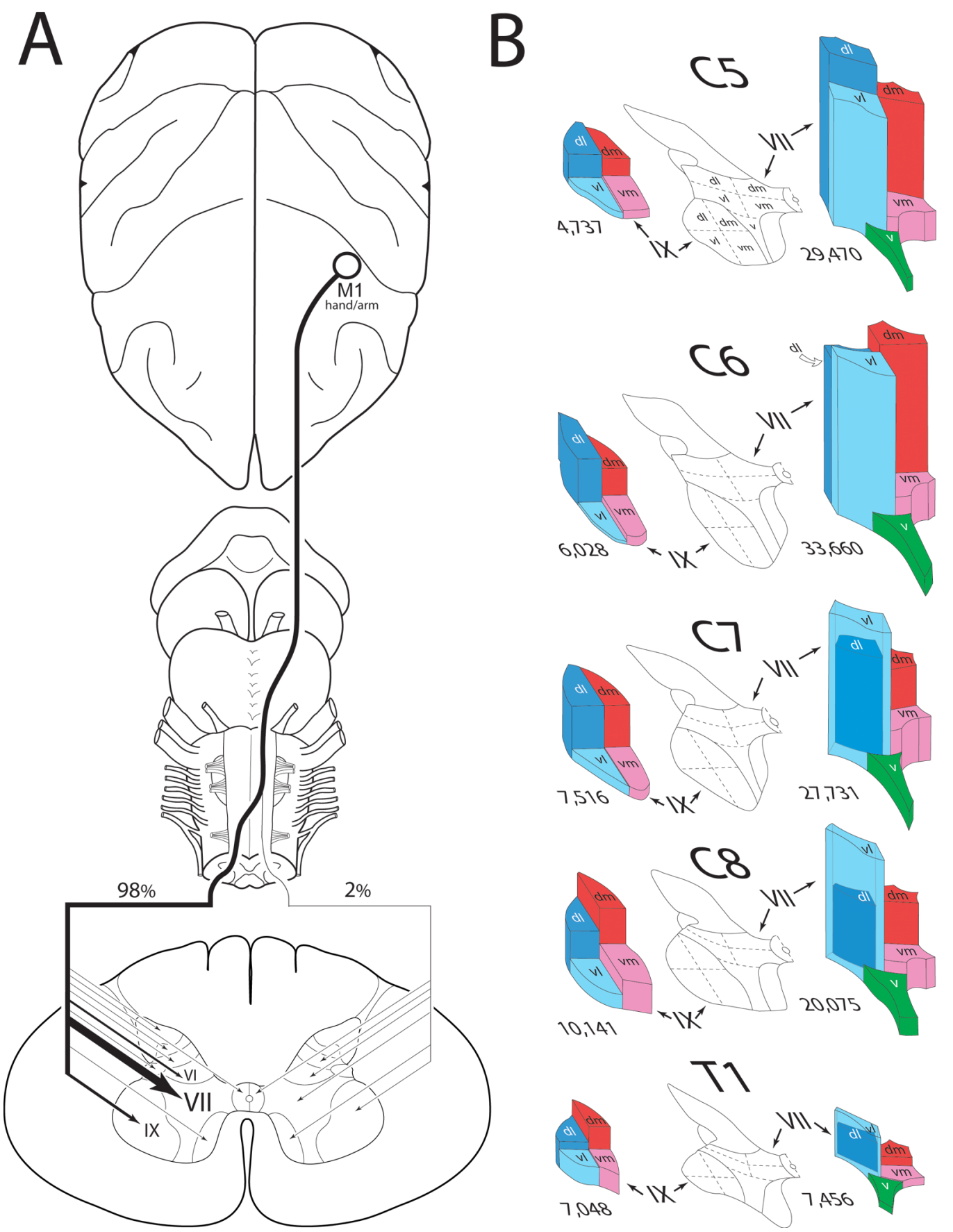

Figure 12.

Summary diagrams illustrating the main findings of the study. The left diagram (A) illustrates the overall corticospinal projection from the hand/arm region of the primary motor cortex (M1). The relative intensity of the projection to spinal cord laminae is indicated by line thickness and arrow head size. Denser terminal projections are represented by increased line thickness and arrow head size. Lighter terminal projections are indicated by progressively thinner lines and arrowheads. The right diagram (B) illustrates in threedimensional format the relative strength of the contralateral projection to the anatomical subsectors of lamina VII and IX at each segmental level. The relative height of each column 
corresponds to the relative number of estimated boutons for each subsector as depicted in Figure 11C (lamina VII) and Figure 11E (lamina IX). The mean number of labeled boutons obtained from the three stereology cases (see Results) is depicted for laminae VII and IX at each cervical level. Note the gradual decrease in the lamina VII projection from rostral to caudal levels and the gradual increase in the lamina IX projection. Also note the projection to lamina VII was consistently most dense in the dorsomedial, dorsolateral and ventrolateral subsectors and the ventrolateral projection was slightly dominant among these, at levels C7$\mathrm{T} 1$. The lamina IX projection was preferentially distributed to the dorsal quadrants at $\mathrm{C} 5-\mathrm{C} 7$ but was more widely dispersed amongst the quadrants at C8 and $\mathrm{T} 1$. 


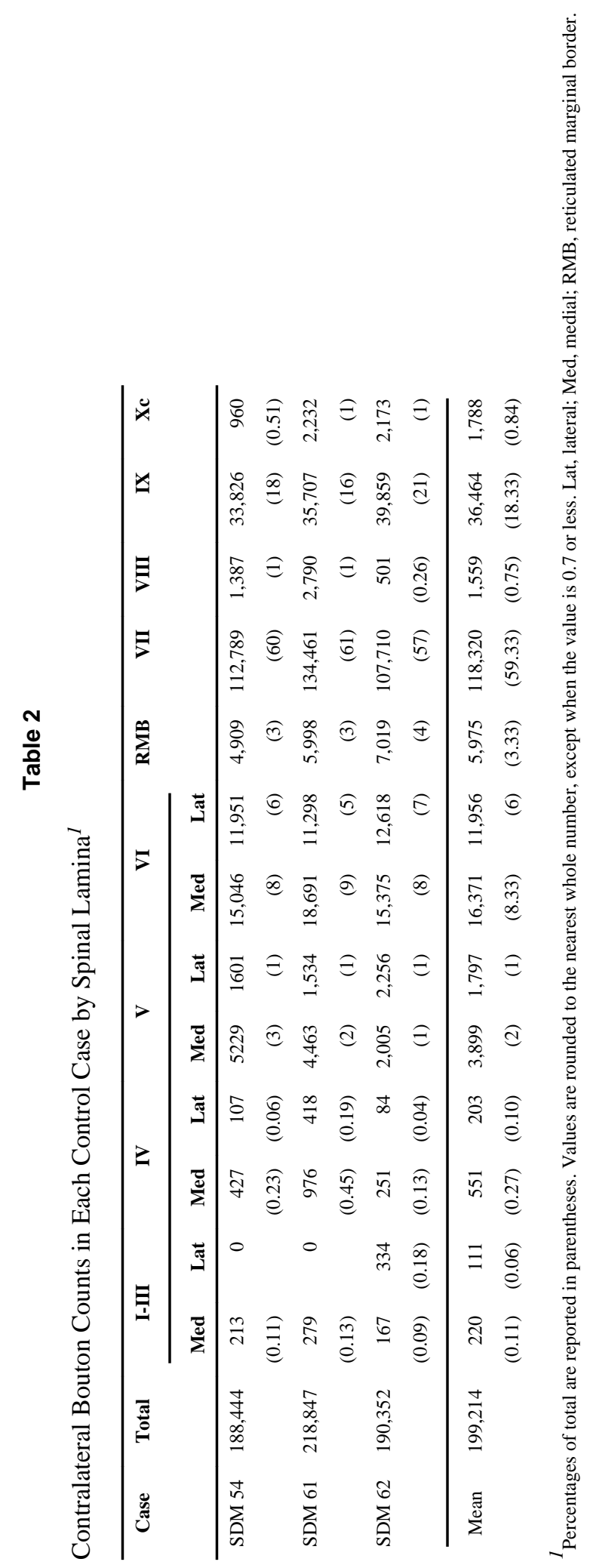




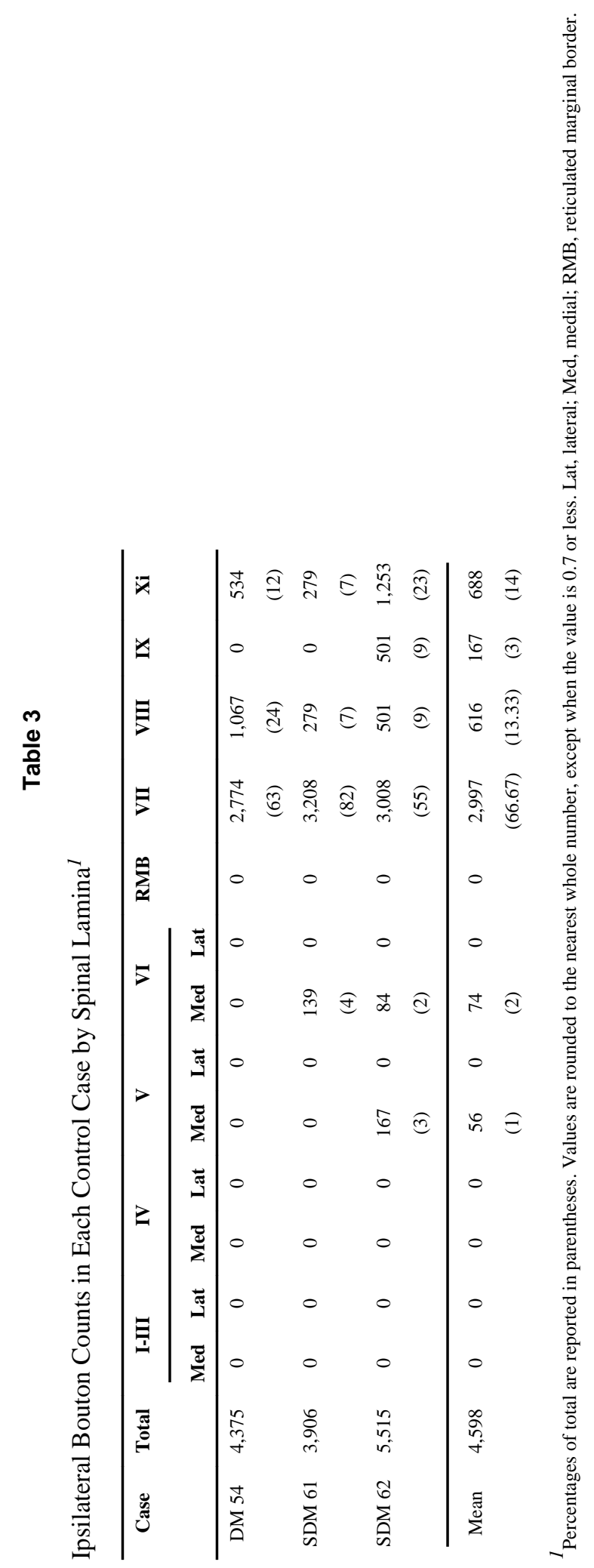

